\title{
Amblyopia Affects the ON Visual Pathway More than the OFF
}

\author{
Carmen Pons, Jianzhong Jin, Reece Mazade, Mitchell Dul, @Qasim Zaidi, and Jose-Manuel Alonso \\ Department of Biological and Visual Sciences, SUNY College of Optometry, New York, New York 10036
}

Visual information reaches the cerebral cortex through parallel ON and OFF pathways that signal the presence of light and dark stimuli in visual scenes. We have previously demonstrated that optical blur reduces visual salience more for light than dark stimuli because it removes the high spatial frequencies from the stimulus, and low spatial frequencies drive weaker ON than OFF cortical responses. Therefore, we hypothesized that sustained optical blur during brain development should weaken ON cortical pathways more than OFF, increasing the dominance of darks in visual perception. Here we provide support for this hypothesis in humans with anisometropic amblyopia who suffered sustained optical blur early after birth in one of the eyes. In addition, we show that the dark dominance in visual perception also increases in strabismic amblyopes that have their vision to high spatial frequencies reduced by mechanisms not associated with optical blur. Together, we show that amblyopia increases visual dark dominance by 3-10 times and that the increase in dark dominance is strongly correlated with amblyopia severity. These results can be replicated with a computational model that uses greater luminance/response saturation in $\mathrm{ON}$ than OFF pathways and, as a consequence, reduces more $\mathrm{ON}$ than OFF cortical responses to stimuli with low spatial frequencies. We conclude that amblyopia affects the $\mathrm{ON}$ cortical pathway more than the OFF, a finding that could have implications for future amblyopia treatments.

Key words: plasticity; receptive field; thalamocortical; thalamus; visual cortex

Significance Statement

Amblyopia is a loss of vision that affects $2-5 \%$ of children across the world and originates from a deficit in visual cortical circuitry. Current models assume that amblyopia affects similarly $\mathrm{ON}$ and OFF visual pathways, which signal light and dark features in visual scenes. Against this current belief, here we demonstrate that amblyopia affects the $\mathrm{ON}$ visual pathway more than the $\mathrm{OFF}$, a finding that could have implications for new amblyopia treatments targeted at strengthening a weak $\mathrm{ON}$ visual pathway.

\section{Introduction}

Amblyopia is a disorder of the visual pathway that causes a loss of visual acuity and contrast sensitivity that is not correctable with prescription lenses. It is the most common cause of vision loss in children (Holmes and Clarke, 2006) and serves as a model to investigate how visual experience modifies cortical wiring. Current models of cortical function assume that amblyopia affects similarly ON and OFF visual cortical pathways (Ding and Sperling, 2006; Huang et al., 2010; Ding et al., 2013; Ding and Levi, 2014). Consequently, visual scientists commonly measure the neuronal deficits of amblyopia with light-dark grating patterns

Received Dec. 21, 2018; revised June 3, 2019; accepted June 5, 2019.

Author contributions: C.P. and J.-M.A. designed research; C.P., J.J., R.M., and J.-M.A. performed research; C.P., J.J., R.M., M.D., Q.Z., and J.-M.A. contributed unpublished reagents/analytic tools; C.P., J.J., R.M., and J.-M.A. analyzed data; C.P. and J.-M.A. wrote the paper.

This work was supported by NIH Grant EY027361.

The authors declare no competing financial interests.

Correspondence should be addressed to Jose-Manuel Alonso at jalonso@sunyopt.edu.

https://doi.org/10.1523/JNEUROSCI.3215-18.2019

Copyright $\odot 2019$ the authors that assume equal cortical responses to lights and darks. In contrast to this assumption, recent evidence indicates that, just as the contralateral eye is better represented than the ipsilateral eye in visual cortex (Wiesel and Hubel, 1963; Adams et al., 2007), the OFF pathway is better represented than the ON pathway (Zemon et al., 1988; Chubb et al., 2004; Jin et al., 2008; Yeh et al., 2009). Moreover, just as monocular deprivation changes the balance between cortical responses from deprived and non-deprived eyes (Wiesel and Hubel, 1963; Hubel et al., 1977), increased exposure to low spatial frequencies (e.g., because of optical blur) changes the ON/OFF response balance making the cortex more OFF dominated (Onat et al., 2011; Kremkow et al., 2014; Pons et al., 2017; Jansen et al., 2019).

Traditionally, ON and OFF visual pathways were thought to remain segregated in thalamus and fully converge in cortex (Hubel and Wiesel, 1962). However, there is increasingly stronger evidence that $\mathrm{ON}$ and OFF pathways remain cortically segregated (McConnell and LeVay, 1984; Norton et al., 1985; Zahs and Stryker, 1988; Jin et al., 2008; Smith et al., 2015; Wang et al., 2015; Kremkow et al., 2016). Moreover, ON and OFF pathways show 
pronounced differences in spatial resolution and response latency that are already present at the level of the retina (Wässle et al., 1981; Dacey and Petersen, 1992; Chichilnisky and Kalmar, 2002) and are further amplified in visual cortex (Jin et al., 2008, 2011a,b; Yeh et al., 2009; Kremkow et al., 2014; Xing et al., 2014; Zurawel et al., 2014; Rekauzke et al., 2016; Jansen et al., 2019; Jimenez et al., 2018). In visual cortex, OFF responses are stronger than ON responses and this cortical OFF dominance has a correlate in human vision and the statistics of natural scenes. Humans are more accurate and faster at seeing static dark targets than light targets (Chubb and Nam, 2000; Chubb et al., 2004; Komban et al., 2011, 2014). Dark features are more abundant than light features in natural scenes (Ratliff et al., 2010) and this dark dominance is most pronounced at low spatial frequencies (Cooper and Norcia, 2015).

Physiological measurements also show that ON cortical responses saturate at lower luminance contrast than OFF cortical responses (Kremkow et al., 2014; Pons et al., 2017) and that this greater response saturation reduces the relative strength of $\mathrm{ON}$ responses to stimuli of low spatial frequency (Onat et al., 2011; Kremkow et al., 2014; Jansen et al., 2019). Therefore, if low spatial frequencies drive weaker ON than OFF cortical responses, sustained optical blur (or any other mechanism that reduces cortical responses to high spatial frequencies) should weaken ON cortical pathways more than OFF during brain development. Consistent with this hypothesis, our results demonstrate that human amblyopia affects vision more for light than dark stimuli.

\section{Materials and Methods}

General psychophysical methods

All visual stimuli were presented on a gamma corrected 24 inch LCD monitor (BenQ XL2420T, $120 \mathrm{~Hz}$, mean luminance: $156 \mathrm{~cd} / \mathrm{m}^{2}$ ) and were generated with Psychtoolbox 3 and custom MATLAB software (MathWorks; Brainard, 1997). The monitor was placed $1 \mathrm{~m}$ in front of the subject's eye and subtended $30.3^{\circ} \times 17.0^{\circ}$ of visual angle $\left(0.016^{\circ} \times\right.$ $0.016^{\circ}$ per pixel). Observers used a chin rest to hold their heads steady in front of the monitor and a numerical keypad to respond in each visual task.

The experiments were conducted in a dark room and performed monocularly (the eye not being tested was covered with a patch). All observers had their vision corrected to achieve the best-possible visual acuity. An observer was initially included in the study ( $n=30$ observers; Table 1$)$ if the best-corrected visual acuity was lower for one eye (amblyopic eye) than the other (fellow eye). Two of these 30 observers were excluded from the analysis because they could not perform the tasks due to very limited vision (E.C.: 20/800 and R.R.: 20/2400). We also excluded a subject that performed poorly in the visual salience task with the fellow eye $(<80 \%)$ probably because of fatigue or poor motivation (Table 1, A.B.). Five of the remaining 27 observers ( 4 tested for visual salience and 5 for grating visual acuity) were classified as near-normal because the difference in visual acuity between the two eyes was smaller than two lines in a Snellen chart (Holmes and Clarke, 2006). The remaining 22 observers were classified as amblyopes and were all included in the data analysis.

We used standard criteria to classify the 22 observers (Holmes and Clarke, 2006) in anisometropic amblyopes (AAs), strabismic amblyopes (SAs), and strabismic-anisometric amblyopes (SAAs). Anisometropic amblyopes had no history of strabismus and had a refraction difference between the eyes large enough to be considered anisometropia (AAs: $n=$ 15 ; see below for criteria). SAs $(n=3)$ had strabismus and no pronounced differences in refraction between the eyes. Some observers had both strabismus and anisometropy and were classified as SAAs $(n=4)$. A difference in refraction between the eyes was considered anisometropic if it was $\geq 1.5$ diopters of hyperopia, $\geq 2$ diopters of myopia, and/or $\geq 2$ diopters of astigmatism (Holmes and Clarke, 2006). If one eye had myopia and the other hyperopia, the refraction difference was considered anisometropic if it was $>1.5$ diopters of spherical equivalent (spherical equivalent $=$ spherical power $+1 / 2$ cylinder power $)$.

We measured dark-light differences in visual salience in 15 of the 22 observers ( 6 females and 9 males, including author J.J.; age range 23-62; Table 1), and dark-light differences in grating visual acuity in 14 of the 22 observers (10 females and 4 males, including author J.J.; age range 18 62; Table 1). We tested both tasks, visual salience and grating visual acuity, in 11 observers. We also tested dark-light differences in grating visual acuity in four control subjects. The near-normal observers were two females and two males, age range $18-55$. The control observers had either 20/20 vision or vision corrected to 20/20 (3 females and 1 male, including two authors, C.P. and J.-M.A.).

The best-corrected visual acuity of the amblyopic observers ranged from $20 / 15$ to $20 / 25$ for the fellow eye and $20 / 30$ to $20 / 400$ for the amblyopic eye. Any observer with unilateral or bilateral ocular disease including early stages of glaucoma was excluded from our sample. We recruited observers with amblyopia through the SUNY College of Optometry University Eye Center. All experiments followed the principles of the Declaration of Helsinki and were approved by the institutional review board at the State University of New York, College of Optometry.

Visual salience. Visual salience was measured monocularly by presenting a randomized sequence of dark and light square targets on a uniform binary-noise background. The observers had to report the number of squares (ranging from one to three) by using a keypad. The probability that the binary-noise-background pixels formed a false target was very small $\left(p=0.5^{36}\right)$ because the side of each square target was six times larger than the side of the background pixels. Nineteen of the 22 observers performed 8 sets of 100 trials in one session ( 800 trials in total). Because of fatigue constrains, the remaining three observers could not perform the same number of trials. Observer M.S. performed 400 trials (four sets of 100 trials) in one session, observer M.F. performed 600 trials ( 6 sets of 100 trials) in one session, and observer K.B. performed 800 trials divided in three sessions administered in three different days $(3,3$, and 2 sets of 100 trials). Every set of 100 trials started with $15 \mathrm{~s}$ adaptation to a midgray screen followed by the first trial. Each subsequent trial started after the observer responded to the previous one. Therefore, the reaction time of each observer determined the duration of each trial. Before starting each trial set, observers took a break of a few minutes. For each observer, we quantified response accuracy and reaction time, separately for the amblyopic and the fellow eye.

Grating visual acuity. We measured grating visual acuity with squarewave gratings that were half-wave rectified. The gratings could be horizontal or vertical, light or dark, and were monocularly presented at different spatial frequencies for $200 \mathrm{~ms}$ followed by a mask that also lasted $200 \mathrm{~ms}$ (the mask was made of overlapping horizontal and vertical gratings). The observers had to report the orientation of the grating in a keypad and, after pressing the keypad, a new grating stimulus was presented after a delay of $250 \mathrm{~ms}$.

Each trial set started with $120 \mathrm{~s}$ of adaptation to either a dark background for light gratings $\left(0.5 \mathrm{~cd} / \mathrm{m}^{2}\right)$ or a light background for dark gratings $\left(312 \mathrm{~cd} / \mathrm{m}^{2}\right)$. Within each trial, the grating and mask had always the same contrast polarity and spatial frequency. Light gratings (312 $\left.\mathrm{cd} / \mathrm{m}^{2}\right)$ were presented on a dark background $\left(0.5 \mathrm{~cd} / \mathrm{m}^{2}\right)$ and dark gratings $\left(0.5 \mathrm{~cd} / \mathrm{m}^{2}\right)$ on a light background $\left(312 \mathrm{~cd} / \mathrm{m}^{2}\right)$. The grating spatial frequency $(0.5,1,2,4,8$, or $16 \mathrm{cpd})$ was changed by modifying the size of the grating while keeping the duty cycle constant (from 6 to $0.2^{\circ}$ per grating side). This approach replicates standard measurements of visual acuity in the eye clinic with Snellen charts (i.e., visual resolution is tested in the eye clinic by reducing the size of Snellen characters). Because the measurements of grating visual acuity are restricted to the high spatial frequency limit of visual resolution, they are not affected by the higher harmonics introduced by the square waves.

We measured grating visual acuity in 14 amblyopic observers (including one author, J.J.), four near-normal observers and four control observers (including two authors, C.P. and J.-M.A.). Every amblyopic observer ran 4800 trials, 2400 with the fellow eye and 2400 with the amblyopic eye. Twelve of the 14 amblyopic observers ran all 4800 trials in one session (2400 trials with the fellow eye and 2400 with the amblyopic eye, 1200 with dark, and 1200 with light gratings for each eye). Two of the 
Table 1. List of observers

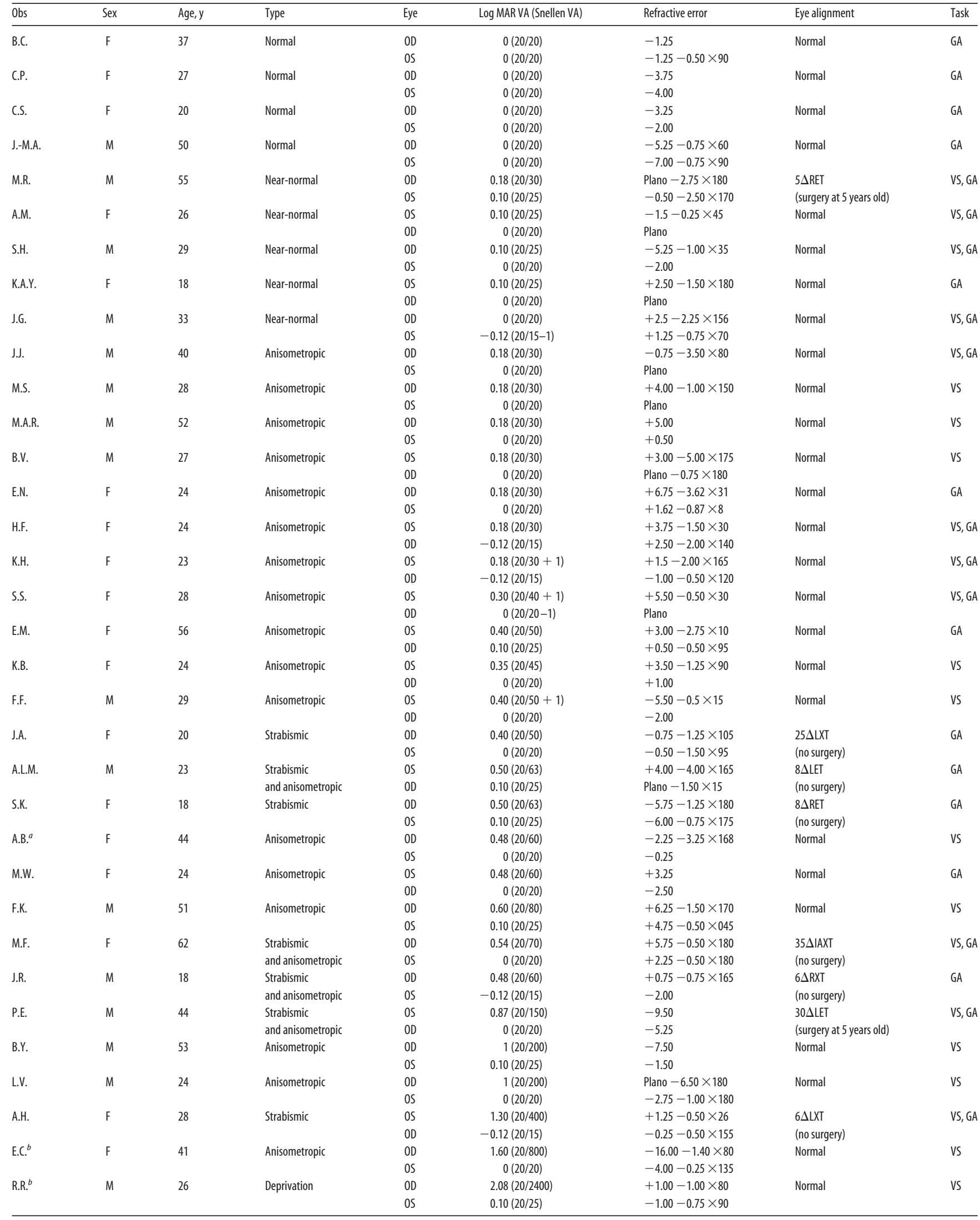

Observers ordered as a function of amblyopia severity, from lowest (top) to highest (bottom). Obs, Observer code; $M$, male; F, female; Eye, type of observer (top, amblyopic; bottom, fellow; $0 D$, oculus dextrus: right eye; OS, oculus sinister: left eye); log MAR, logarithm of the minimum angle of resolution; Snellen VA, visual acuity measured with Snellen chart; refractive error: spherical power, cylinder power, and axis; normal, eyes aligned; RET, right esotropia; LET, left esotropia; LXT, left exotropia; IAXT intermittent alternating exotropia; VS, visual salience; GA, grating acuity).

${ }^{a}$ Excluded from the analysis because the best performance with fellow eye was $<90 \%$.

${ }^{b}$ Excluded from the analysis because the observers could not perform the tasks because of their very limited vision. 
17 amblyopic observers (J.J. and K.H.) ran the 4800 trials in two sessions (600 for light and 600 for dark gratings for each eye in one session, 2400 trials per session). Control observers ran 2400 trials with one eye.

Data acquisition and analysis. Reaction time histograms were plotted using a $0.05 \mathrm{~s}$ bin and shown as raw data. Statistical significance was assessed with Wilcoxon tests. For all tests, the level of significance was marked as follows: ${ }^{*} p<0.05,{ }^{* *} p<0.01,{ }^{* *} p<0.001$. All error bars show SEM.

\section{Perceptual model}

To simulate the amblyopia deficits reported in this paper, we used a computational model that incorporates a greater luminance/response saturation within the ON than OFF cortical pathways, as demonstrated experimentally (Kremkow et al., 2014; Pons et al., 2017). We call this ON luminance/response saturation neuronal blur because it blurs stimuli through neuronal responses, not optics. The model is able to reproduce the development of amblyopia, the psychophysical deficits that we report, and deficits in cortical response suppression demonstrated in amblyopic macaques (Shooner et al., 2017).

The model has four stages: input, retinal, thalamocortical, and cortical stage (see Fig. $7 A-D$ ). At the input stage, we feed images of the visual stimuli to the model and blur the images with a Gaussian filter to simulate the optical blur of the eye, which is equal for lights and darks. At the retinal stage, we blur the images with the luminance/response functions of ON and OFF retinal pathways to simulate the neuronal blur, which is larger for lights than darks because of the greater luminance response saturation of the ON pathway (Kremkow et al., 2014; Pons et al., 2017). At the thalamocortical stage, we convolve the blurred images with centersurround receptive fields. Finally, at the cortical stage, we multiply the result of the convolution by the synaptic weights of ON and OFF pathways from the fellow and amblyopic eyes, we add noise, and we combine the pathways from the two eyes.

This general model is able to simulate the development of amblyopia (development model variation), ON-OFF differences in psychophysical deficits (psychophysics model variation) and deficits in cortical response suppression (physiology model variation). In the developmental model variation, the only difference between amblyopic and the fellow eyes is the amount of optical blur at the input stage (larger for the amblyopic eye), when modeling anisometropic amblyopia. When modeling strabismic amblyopia, the only difference between the amblyopic and fellow eyes is the receptive field size and cortical noise (larger for the amblyopic eye). In the psychophysics and physiology variations, the only difference between the amblyopic and the fellow eyes is the synaptic weights at the cortical stage (stronger for the fellow eye). Below, we provide a detailed description of the general stages of the model and model variations.

General stages of the model. At the input stage, we feed the model with images of the visual stimuli such as square targets embedded in noise, gratings, or noise masks. The image of the stimuli, I $(x, y)$, is first convolved with a Gaussian filter that has a standard deviation (SD) of $0.5 \mathrm{~min}$ of arc. This convolution simulates the optical blur caused by the optical point-spread-function of the eye (PSF; Campbell and Gubisch, 1966). The point-spread-function is normal in the fellow $\left(\mathrm{PSF}^{\mathrm{F}}\right)$ and amblyopic $\left(\mathrm{PSF}^{\mathrm{A}}\right)$ eyes of adults with proper optical correction $\left(\mathrm{PSF}^{\mathrm{A}}=\mathrm{PSF}^{\mathrm{F}}\right)$, but larger than normal in the amblyopic eye of an infant developing anisometropic amblyopia $\left(\mathrm{PSF}^{\mathrm{A}}>\mathrm{PSF}^{\mathrm{F}}\right)$. After applying optical blur, the resulting luminance of each image pixel is $\mathrm{L}^{\mathrm{F}}(x, y)$ for the fellow eye and $\mathrm{L}^{\mathrm{A}}(x, y)$ for the amblyopic eye, as shown in Equation 1.

$$
\begin{aligned}
& L^{F}(x, y)=P S F^{F}(x, y) \circledast I(x, y) \\
& L^{A}(x, y)=P S F^{A}(x, y) \circledast I(x, y)
\end{aligned}
$$

At the retinal stage, we simulate $\mathrm{ON}$ and $\mathrm{OFF}$ bipolar-cell responses $\left(\mathrm{B}_{\mathrm{ON}}\right.$ and $\left.\mathrm{B}_{\mathrm{OFF}}\right)$ and apply neuronal blur to $\mathrm{L}(x, y)$ by using Naka-Rushton functions with larger luminance/response saturation for ON than OFF retinal pathways, as shown in Equation 2a (same equation for both eyes). OFF responses are modeled as the absolute value of the Naka-Rushton function minus one to simulate the sign reversal at the OFF bipolar cell; low values in $\mathrm{L}(x, y)$ drive large values of $\mathrm{B}_{\mathrm{OFF}}(x, y)$, and large values of $\mathrm{L}(x, y)$ drive low values of $\mathrm{B}_{\mathrm{OFF}}(x, y)$. In the Naka-Rushton functions, the exponent $(n)$ and the luminance that generates $50 \%$ of the maximum response $\left(\mathrm{L}_{50}\right)$ determine the amount of neuronal blur (i.e., lower values cause greater saturation in the luminance/response function and greater neuronal blur). Both the exponent and $\mathrm{L}_{50}$ are larger for OFF than ON pathways $\left(n=1.6\right.$ for ON and 2.5 for OFF, $\mathrm{L}_{50}=0.1$ for ON and 0.5 for $\mathrm{OFF}$ ). The parameters of neuronal blur are the same for all model variations. We also normalize the $\mathrm{B}_{\mathrm{ON}}$ and $\mathrm{B}_{\mathrm{OFF}}$ responses (divide by maximum response across all $x, y$ pixel values) to make $\mathrm{ON}$ and $\mathrm{OFF}$ maximum responses equal at the retinal stage, as shown in Equation $2 \mathrm{~b}$.

$$
\begin{gathered}
B_{O N}(x, y)=\frac{L(x, y)^{n}}{L_{50}^{n}+L(x, y)^{n}} \\
B_{O F F}(x, y)=\left|\frac{L(x, y)^{n}}{L_{50}^{n}+L(x, y)^{n}}-1\right| \\
B_{O N}(x, y)=\frac{B_{O N}(x, y)}{\max \left(B_{O N}\right)} \\
B_{O F F}(x, y)=\frac{B_{O F F}(x, y)}{\max \left(B_{O F F}\right)}
\end{gathered}
$$

At the thalamocortical stage, we convolve the $\mathrm{B}_{\mathrm{ON}}$ and $\mathrm{B}_{\mathrm{OFF}}$ responses with $\mathrm{ON}$ and $\mathrm{OFF}$ center-surround receptive fields, $\mathrm{RF}_{\mathrm{ON}}$ and $\mathrm{RF}_{\mathrm{OFF}}$, as shown in Equation 3. This stage combines in one single operation all retinal and thalamic processing by center-surround receptive fields (e.g., bipolar cells, retinal ganglion cells, and thalamic cells). We model the center-surround receptive field as a difference-of-Gaussians function, with the surround being three times larger and five times weaker than the center for all simulations and model variations.

The result of the convolution at the thalamocortical stage is measured at the most relevant image region for each task $\left(x_{i-j}, y_{i-j}\right)$, where $i-j$ represent the range of pixel values within $x$ and $y$ image coordinates. The selected region of the convolution $\left(x_{i-j}, y_{i-j}\right)$, is at the center of light/dark targets for the visual salience task, the gap between grating bars for the grating acuity task, and the center of the stimulus for the measurements of cortical response suppression. The selection of this region is based on the assumption that the receptive field population used to detect the targets in the salience task is aligned with the target center, the receptive field population used to discriminate a gap between two bars is aligned with the bar gap and the receptive field population that drives interocular suppression is aligned with the center of the stimuli presented to each eye.

The thalamocortical response is calculated as the subtraction between the maximum and minimum value of the convolution at the selected region, separately for $\mathrm{ON}\left(\mathrm{T}_{\mathrm{ON}}\right)$ and OFF pathways $\left(\mathrm{T}_{\mathrm{OFF}}\right)$, as shown in Equation 3. This subtraction makes the thalamocortical response zero when there is no stimulus contrast.

$$
\begin{aligned}
T_{O N}\left(x_{i-j}, y_{i-j}\right)=\max \left(B_{O N}\left(x_{i-j}, y_{i-j}\right) \circledast\right. & \left.R F_{O N}\right) \\
- & \min \left(B_{O N}\left(x_{i-j}, y_{i-j}\right) \circledast R F_{O N}\right) \\
T_{O F F}\left(x_{i-j}, y_{i-j}\right)=\max \left(B_{O F F}\left(x_{i-j}, y_{i-j}\right)\right. & \left.\circledast R F_{O F F}\right) \\
& -\min \left(B_{O F F}\left(x_{i-j}, y_{i-j}\right) \circledast R F_{O F F}\right)
\end{aligned}
$$

At the cortical stage, we multiply the responses from each thalamocortical pathway by a gain factor that simulates the synaptic weights $\left(W_{O N}^{F}\right.$, $\left.W_{O F F}^{F}, W_{O N}^{A}, W_{O N}^{A}\right)$, as shown in Equation 4 . This equation generates the cortical responses for ON and OFF pathways from fellow and amblyopic eyes $\left(C_{O N}^{F}, C_{O F F}^{F}, C_{O N}^{A}, C_{O N}^{A}\right)$, which will be used as inputs in the different model variations.

$$
\begin{aligned}
& C_{O N}^{F}=W_{O N}^{F} * T_{O N}^{F} \\
& C_{O F F}^{F}=W_{O F F}^{F} * T_{O F F}^{F} \\
& C_{O N}^{A}=W_{O N}^{A} * T_{O N}^{A} \\
& C_{O F F}^{A}=W_{O F F}^{A} * T_{O F F}^{A}
\end{aligned}
$$

Developmental model variation. In the developmental model variation, we run different simulations for anisometropic and strabismic amblyopes. To simulate anisometropic amblyopia, we first run the general model with dark and light grating stimuli using larger optical blur for the amblyopic eye than the fellow eye. To simulate strabismic amblyopia, we 
use the same optical blur for both eyes but make the receptive field larger and the responses noisier for the amblyopic than the fellow eye. The larger receptive field and noisier responses simulate the lower spatial resolution and lower signal-to-noise of the cortical network sampling the stimulus in strabismic amblyopia (Kiorpes et al., 1998).

The cortical responses $\left(C_{O N}^{F}, C_{O F F}^{F}, C_{O N}^{A}, C_{O N}^{A}\right)$ are multiplied by a Gaussian function $(G)$ to simulate the peristimulus time histogram of the visual responses from each cortical pathway. They are also normalized by the maximum response across the four cortical pathways $\left(C_{\max }\right)$, as shown in Equation 5.

$$
\begin{aligned}
& C_{O N}^{F}=G * \frac{C_{O N}^{F}}{C_{\max }} \\
& C_{O F F}^{F}=G * \frac{C_{O F F}^{F}}{C_{\max }} \\
& C_{O N}^{A}=G * \frac{C_{O N}^{A}}{C_{\max }} \\
& C_{O F F}^{A}=G * \frac{C_{O F F}^{A}}{C_{m a x}} \\
& C_{\max }=\max \left(\left[C_{O N}^{F}, C_{O F F}^{F}, C_{O N}^{A}, C_{O F F}^{A}\right]\right)
\end{aligned}
$$

At each developmental step (DS) of the developmental model, we subtract the cortical responses between eyes while using a multiplicative factor on the subtractive term to simulate the strength of interocular suppression ( $\mathrm{S}$; strength of response suppression from one eye by the other). We also multiply the cortical response from each eye by a synaptic weight that is equal to 1 at the first developmental step and then changes during development (e.g., $W_{O N}^{A}$ for the ON pathway of the fellow eye). We then add random noise $(\mathrm{N})$, and limit the cortical response to a maximum value of 1, as shown in Equation 6.

$$
\begin{gathered}
C_{O N}^{F}=W_{O N}^{F} * C_{O N}^{F}-W_{O N}^{A} * C_{O N}^{A} * S+N\left\{\begin{array}{l}
\text { if } C_{O N}^{F}<1, \\
\text { otherwise } C_{O N}^{F}=1
\end{array}\right\} \\
C_{O F F}^{F}=W_{O F F}^{F} * C_{O F F}^{F}-W_{O F F}^{A} * C_{O F F}^{A} * S+N\left\{\begin{array}{l}
\text { if } C_{O F F}^{F}<1, \\
\text { otherwise } C_{O F F}^{F}=1
\end{array}\right\} \\
C_{O N}^{A}=W_{O N}^{A} * C_{O N}^{A}-W_{O N}^{F} * C_{O N}^{F} * S+N\left\{\begin{array}{l}
\text { if } C_{O N}^{A}<1, \\
\text { otherwise } C_{O N}^{A}=1
\end{array}\right\} \\
C_{O F F}^{A}=W_{O F F}^{A} * C_{O F F}^{A}-W_{O F F}^{F} * C_{O F F}^{F} * S+N\left\{\begin{array}{l}
\text { if } C_{O F F}^{A}<1, \\
\text { otherwise } C_{O F F}^{A}=1
\end{array}\right\}
\end{gathered}
$$

As amblyopia progresses at each DS, the synaptic pruning $\left(\mathrm{SP}_{\mathrm{DS}}\right)$ reduces the synaptic weight of each cortical pathway if the maximum cortical response $\left(C_{O N}^{F}\right)$ is lower than a minimum response $\left(R_{\min }\right)$, as shown in Equation $7 \mathrm{a}$ for the ON pathway from the fellow eye, $W_{O N_{D S}}^{A}$ (same for all pathways). The $\mathrm{SP}_{\mathrm{DS}}$ decreases at each developmental step through a pruning reduction factor $(\mathrm{PR})$ multiplied by the $\mathrm{DS}$ counter. The $\mathrm{SP}_{\mathrm{DS}}$ remains effective while it is larger than 1 and until the developmental step counter reaches the end of the critical period (ECP). The synaptic pruning is identical for all pathways (illustrated for $W_{O N}^{F}$ in Eq. 7).

$$
\begin{gathered}
W_{O N_{D S}}^{F}=\frac{W_{O N_{D S-1}}^{F}}{S P_{D S}}\left\{\text { if } \max \left(C_{O N}^{F}\right)<R_{\min }\right. \\
S P_{D S}=S P_{D S-1}-P R * D S\left\{\begin{array}{l}
\text { if } S P_{D S}>1 \\
\text { if } D S<E C P
\end{array}\right.
\end{gathered}
$$

The outputs of the developmental model are the synaptic weights for ON and OFF cortical pathways from the amblyopic eye and fellow eyes $\left(W_{O N}^{F}\right.$, $\left.W_{O F F}^{F}, W_{O N}^{A}, W_{O N}^{A}\right)$, which can range from 0 to 1 . The simulations generate weaker synaptic weights for the amblyopic than the fellow eye and weaker synaptic weights for the ON than OFF pathways within the amblyopic eye. As an example, the model outputs $W_{O F F}^{F}=1, W_{O N}^{F}=1, W_{O F F}^{A}=0.92$, and $W_{O N}^{A}=0.83$ when we use the following parameters: receptive field size $=4$ pixels ( $1 \mathrm{~min}$ of arc), grating stimulus $=6$ cycles per degree, optical blur $=0.5 \mathrm{~min}$ of arc for the fellow eye and $1 \mathrm{~min}$ of arc for the amblyopic eye, noise level $=0.1 \pm 0.06, \mathrm{ECP}=30$ developmental steps, $\mathrm{S}=0.2$, minimum response $\left(R_{\min }\right)=0.68$, initial $\mathrm{SP}=1.0075(0.75 \%$ decrease per DS), $\mathrm{PR}=0.000005$ ( $0.0005 \%$ reduction per DS).

Psychophysics model variation. In the psychophysics model variation, we first run the general model with dark and light stimuli using the synaptic weights generated by the developmental model variation. We then simulate the cortical responses for each pathway using similar equations to those described above. We first multiply the cortical responses from the general model with a Gaussian function $(G)$ to simulate the peristimulus time histogram for the visual responses from each cortical pathway, as shown in Equation 8.

$$
\begin{aligned}
& C_{O N}^{F}=G * C_{O N}^{F} \\
& C_{O F F}^{F}=G * C_{O F F}^{F} \\
& C_{O N}^{A}=G * C_{O N}^{A} \\
& C_{O F F}^{A}=G * C_{O F F}^{A}
\end{aligned}
$$

We then subtract the cortical responses between eyes using a multiplicative factor on the subtractive term to simulate the strength of the interocular suppression $(\mathrm{S})$, and add random noise $(\mathrm{N})$, as shown in Equation 9. We also add a subtractive term to simulate luminance inhibition (I), a noise reduction dependent on background luminance (Xing et al., 2014). This luminance inhibition is important to simulate deficits in cortical suppression (described below) but plays no role in simulating psychophysical deficits. Therefore, we could use $N$ instead of $N-$ I in Equation 9 and the psychophysical deficits would be the same. We use $N-$ I just for consistency across model variations.

$$
\begin{array}{cl}
C_{O N}^{F}=C_{O N}^{F}-C_{O N}^{A} * S+N-I & \left.\begin{array}{l}
\text { if } C_{O N}^{F}<1, \\
\text { otherwise } C_{O N}^{F}=1
\end{array}\right\} \\
C_{O F F}^{F}=C_{O F F}^{F}-C_{O F F}^{A} * S+N-I \quad\left\{\begin{array}{l}
\text { if } C_{O F F}^{F}<1, \\
\text { otherwise } C_{O F F}^{F}=1
\end{array}\right\} \\
C_{O N}^{A}=C_{O N}^{A}-C_{O N}^{F} * S+N-I \quad\left\{\begin{array}{l}
\text { if } C_{O N}^{A}<1, \\
\text { otherwise } C_{O N}^{A}=1
\end{array}\right\} \\
C_{O F F}^{A}=C_{O F F}^{A}-C_{O F F}^{F} * S+N-I \quad\left\{\begin{array}{l}
\text { if } C_{O F F}^{A}<1, \\
\text { otherwise } C_{O F F}^{A}=1
\end{array}\right\}
\end{array}
$$

We simulate psychophysical performance by averaging the cortical responses measured in multiple trials ( $n$ trials, from trial 1 to trial $n$ ), as shown in Equation 10 for the ON pathway from the fellow eye (same equation for all pathways). We set the perceptual average threshold at 1 to make the average probability of correct responses equal to the average cortical response in most trials. When the average cortical response is larger than the perceptual threshold, the average probability of a correct response reaches its maximum value, which is 1 .

$$
P_{O N}^{F}=\frac{\sum_{1}^{n} C_{O N_{t}}^{F}}{n}\left\{\begin{array}{l}
\text { if } P_{O N}^{F}<1, \\
\text { otherwise, } P_{O N}^{F}=1
\end{array}\right\}
$$

To simulate our psychophysical results, we use a receptive field size of 4 pixels for grating visual acuity ( 1 min of arc) and 8 pixels for visual salience ( $2 \mathrm{~min}$ of arc). The absolute values of receptive field size are not realistic and were arbitrarily chosen to make the simulations simpler. We also use slightly weaker synaptic weights for grating visual acuity $\left(W_{O F F}^{F}=1, W_{O N}^{F}=1, W_{O F F}^{A}=0.92\right.$, and $\left.W_{O N}^{A}=0.8\right)$ than for visual salience $\left(W_{O F F}^{F}=1, W_{O N}^{F}=1, W_{O F F}^{A}=0.92\right.$, and $\left.W_{O N}^{A}=0.85\right)$ because the neuronal populations recruited by the two tasks are likely to be different and differently affected by amblyopia. For example, the discrimination of narrow bar gaps in grating visual acuity should use neurons with smaller receptive fields than the detection of large targets in visual salience. Optical blur during amblyopia development should also affect more the neurons that have small receptive fields. The neuronal noise was set to $0.05 \pm 0.03$ for visual salience and $0.07 \pm 0.05$ for grating visual acuity. The other parameters were the same for the two visual tasks. The luminance inhibition was 0.15 , the number of trials was 50 , and the 
strength of intraocular suppression was 0 because all tests were monocular (the fellow eye was always covered with a patch).

Physiology model variation. In the physiology model variation, we run the general model with three different stimuli: a grating of 7 cycles $/{ }^{\circ}$, a noise stimulus with mean luminance and mean SD of 0.5 , and a gray screen also with a mean luminance of 0.5 (image luminance ranging from 0 to 1 ). We multiply the cortical responses (e.g., $C_{O N_{\text {grating }}}^{F}, C_{O F F_{\text {grating }}}^{F}$, $C_{O N_{\text {grating }}}^{A}, C_{O N_{\text {grating }}}^{A}$ by Gaussian functions $(G)$ to generate the peristimulus time histogram for each single neuron. We then add random noise $(\mathrm{N})$ and a subtractive luminance inhibition (I) as in Equation 9. In the physiological variation, we simulate multiple neurons with different response amplitudes. Therefore, we multiply all terms by a neuronal response range $(R)$ that generates variable response amplitudes across neurons (from 0 to a maximum response value). We then rectify the response so that the minimal value is always 0, as shown in Equation 11 for $C_{O N}^{F}$ (same equations for the other pathways).

$$
\begin{aligned}
& C_{O N_{\text {grating }}}^{F}=R *\left(G * C_{O N_{\text {grating }}}^{F}+N-I\right) \quad\left\{\begin{array}{l}
\text { if } C_{O N_{\text {grating }}}^{F}>0 \\
\text { otherwise, } C_{O N_{\text {grating }}}^{F}=0
\end{array}\right\} \\
& C_{O N_{\text {noise }}}^{F}=R *\left(G * C_{O N_{\text {noise }}}^{F}+N-I\right) \quad\left\{\begin{array}{l}
\text { if } C_{O N_{\text {noie }}}^{F}>0 \\
\text { otherwise, } C_{O N_{\text {noise }}}^{F}=0
\end{array}\right\} \\
& C_{O N_{g r a y}}^{F}=R *\left(G * C_{O N_{g r a y}}^{F}+N-I\right) \quad\left\{\begin{array}{l}
\text { if } C_{O N_{\text {gray }}}^{F}>0 \\
\text { otherwise, } C_{O N_{\text {gray }}}^{F}=0
\end{array}\right\}
\end{aligned}
$$

In the second stage of this physiological variation, we subtract the cortical responses between eyes using a multiplicative factor on the subtractive term to simulate the strength of interocular suppression ( $\mathrm{S}$ ), as shown in Equation 12.

$$
C_{O N_{\text {grating-noise }}}^{F-A}=C_{O N_{\text {grating }}}^{F}-S * C_{O N_{\text {noise }}}^{A}
$$

The physiological model variation simulates the deficits in cortical response suppression demonstrated in macaques with binocular stimuli (Shooner et al., 2017). In these simulations, we use 50 neurons with a receptive field size of 3 pixels and weaker synaptic weights for ON than OFF pathways from the amblyopic eye $\left(W_{O F F}^{F}=1, W_{O N}^{F}=1, W_{O F F}^{A}=0.92\right.$, and $\left.W_{O N}^{A}=0.85\right)$. To match the response values reported in the physiological experiments (Shooner et al., 2017), we use a neuronal response range between 0 and 2.3 (similar to the neuronal range reported by Shooner et al., 2017, their Fig. 2), a neuronal noise of $0.05 \pm 0.03$ and the maximum interocular suppression $(\mathrm{S}=1)$. The luminance inhibition (I) is 0.9 for all the stimuli and cortical pathways except for the ON pathway from the amblyopic eye that has a luminance inhibition of 0.4. This reduction in luminance inhibition is needed to simulate the physiological results and is consistent with the greater deficits for ON than OFF pathways that we report. This luminance inhibition is only measurable when using large homogeneous surfaces (Xing et al., 2014); therefore, we apply it only to gray backgrounds.

\section{Results}

Humans are born with immature eyes that cannot properly focus images on the retina. The optical blur resulting from defocus reduces the high spatial frequencies of the retinal image and makes low spatial frequencies the main drivers of visual cortical activity early after birth. As the eye starts growing and maturing, the optical blur decreases and the high spatial frequencies start driving cortical responses. However, in some children, one eye remains out of focus for a longer time and becomes amblyopic (i.e., a loss of visual acuity that is not correctable with lenses). Reliable measures of visual acuity are difficult to obtain in young children; therefore, a young eye can remain out of focus for several years. Because optical blur reduces the stimulus high spatial frequencies, and low spatial frequencies drive weaker ON than OFF cortical responses (Onat et al., 2011; Kremkow et al., 2014; Pons et al., 2017; Jansen et al., 2019), we predicted that sustained optical blur should weaken ON cortical pathways more than OFF during brain development (Fig. 1A). In turn, the weaker $\mathrm{ON}$ pathway should make it more difficult for anisometropic amblyopes to see light than dark stimuli. We also predicted that, as amblyopia becomes more severe, the dark-light differences should still be present when discriminating large targets (Fig. 1B) but not when discriminating the narrow gaps of a grating, which are no longer visible (Fig. 1C). The results below provide strong support for these predictions.

\section{Amblyopia affects visual salience of light stimuli more than dark stimuli}

We measured visual salience for dark and light stimuli with a task that we have used extensively in the past (Komban et al., 2011; Wool et al., 2015; Zhao et al., 2015; Pons et al., 2017). In this visual task, we monocularly presented one to three light or dark squares in a binary noise background (mean luminance: $156 \mathrm{~cd}$ / $\mathrm{m}^{2}$ ) and asked participants to report as fast as possible the number of square targets (Fig. $2 A$ ). We have previously demonstrated that humans with normal vision are faster at reporting the number of dark than light stimuli in this task (Komban et al., 2011; Zhao et al., 2015; Pons et al., 2017) and we now report the same finding for the fellow eye of amblyopic observers (Fig. 2B-D). Consistent with previous results, the reaction times were consistently faster for darks than lights in each of the 100 trial sets performed by the same observer (Fig. $2 B$; notice the tendency for the red lines to be above the blue lines in all trial sets). Moreover, this trend remained present even when the observers started responding faster as they became more familiar with the task (Fig. $2 B$; notice faster reaction times for trial set 8 than 1$)$. The distribution of reaction times peaked at longer values for lights than darks (Fig. 2C) but the distribution area could be similar (Fig. 2C; notice the logarithmic scale of the $x$-axis). Consequently, the average reaction time was faster for darks than lights (Fig. 2D, right) even if there were no significant differences in accuracy measured as the percentage of correct trials (Fig. 2D, left).

When performing the visual salience task, the fellow eye of amblyopic observers behaved similarly to the eye of a normal observer. As with normal (Komban et al., 2011; Zhao et al., 2015; Pons et al., 2017) or near-normal eyes (Fig. 3A-C), the fellow eyes of amblyopic observers revealed dark-light differences in visual salience more pronounced for reaction time than accuracy (Fig. $3 D-F)$. On average, the reaction time with the fellow eye was $41 \%$ faster for darks than lights (Fig. $3 G-I ; 1.78 \pm 0.02 \mathrm{~s}$ for darks vs $3.04 \pm 0.03 \mathrm{~s}$ for lights, $p<0.001$, Wilcoxon test). However, the accuracy with the fellow eye was just $5 \%$ higher for darks than lights (Fig. $3 G-I ; 95.72 \pm 0.36 \%$ for darks vs $90.90 \pm 0.67 \%$ for lights, $p<0.001$, Wilcoxon test). The small dark-light differences in accuracy for the fellow eye were independent of amblyopia severity, which ranged from a 0.18 (Fig. 3D-F, top) to a $1.42 \mathrm{log}$ MAR deficit (Fig. 3D-F, bottom). Therefore, we used the fellow eye as an internal control of normal dark-light differences for each amblyopic observer.

Unlike for the fellow eye, dark-light differences in accuracy for the amblyopic eye were very pronounced, particularly in observers with pronounced visual acuity loss (Fig. $3 D-F$ ). On average, dark-light differences in accuracy were 3 times larger for the amblyopic eye than the fellow eye (Fig. $3 G-I ; 14.47 \pm 1.18 \%$ vs $4.82 \pm 0.57 \%, p<0.001$, Wilcoxon test) and dark-light differences in reaction time were 1.3 times larger for the amblyopic than fellow eye (Fig. $3 G-I ; 1.61 \pm 0.12 \mathrm{~s}$ vs $1.26 \pm 0.07 \mathrm{~s}, p=$ 0.004 , Wilcoxon test). The differences in reaction time could be absent in observers with severe amblyopia (Fig. 3D-F, bottom, observer A.H.) because these observers could not discriminate 
A Physical stimulus
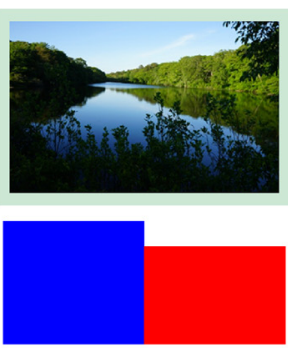

OFF

ON
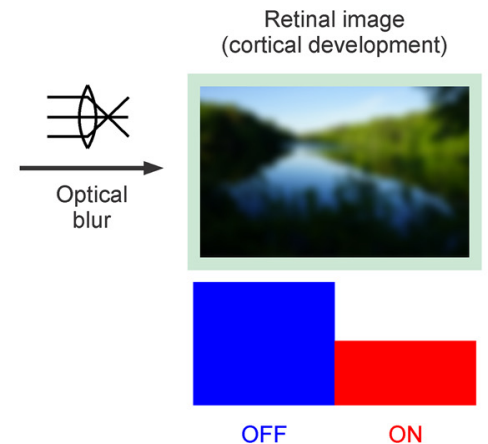

OFF

ON
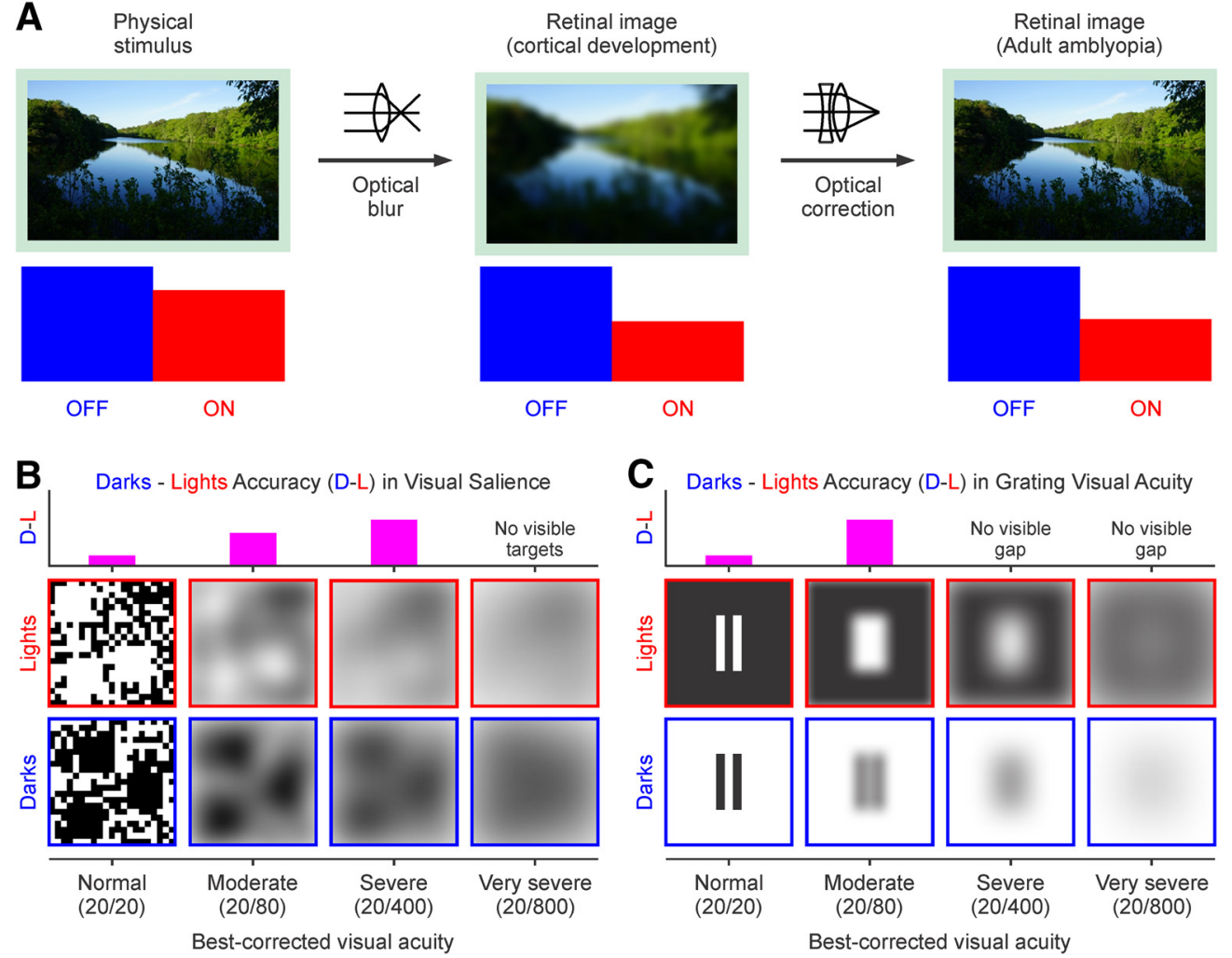

Figure 1. Predicted effect of optical blur on the development of ON/OFF response balance in visual cortex. $A$, Top, An image seen at focus (left), after introducing optical blur (middle), and after correcting the optical blur with a lens (right). Bottom, We hypothesize that sustained optical blur during development weakens ON cortical pathways (red) more than OFF (blue), and that this change in ON/OFF response balance remains in adults even after correcting the optical blur (right). $\boldsymbol{B}$, Top, We hypothesized that dark-light differences in visual salience increase with amblyopia in observers with mild to severe loss of vision (0.18-1.3 logMAR visual acuity loss). Bottom, Diagram illustrating how different levels of amblyopia would affect the stimuli. $\boldsymbol{C}$, Top, We hypothesized that dark-light differences in grating visual acuity can only be revealed in observers with mild to moderate amblyopia (0.18 - 0.6 logMAR visual acuity loss) because observers with severe amblyopia cannot see the gap between the bars. Bottom, Same as $\boldsymbol{B}$ but for the grating visual acuity task stimuli.

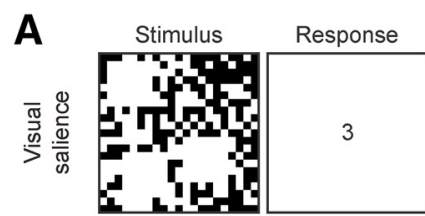

B

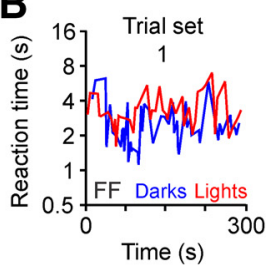

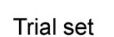

Trial set

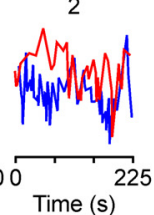

Stimulus
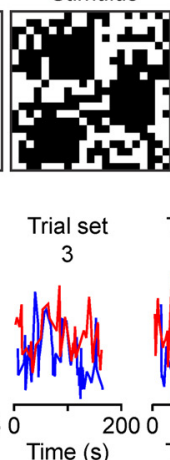

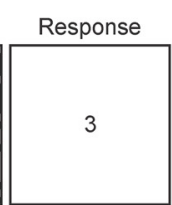

Trial se

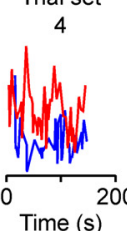

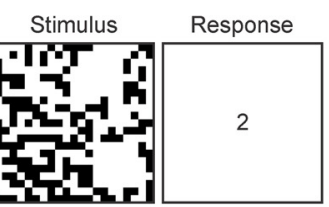

Trialset Trialset Trialset

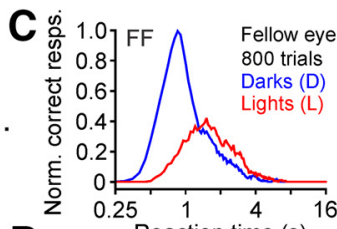

D

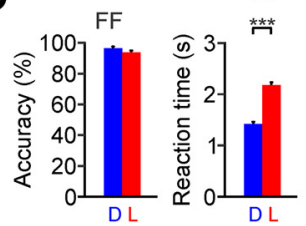

Figure 2. Dark-light asymmetries in visual salience for the fellow eye of a human observer with amblyopia. $\boldsymbol{A}$, We presented 1-3 light or dark squares in a noisy background. Observers had to report as fast and as accurately as possible the number of squares (correct responses shown in squares next to stimulus). $\boldsymbol{B}$, Sequence of reaction times for correct answers from amblyopic subject F.F. (fellow eye, 8 sets of 100 trials). The $y$-axis shows the average reaction time for consecutive trial sets and the $x$-axis the time intervals between trials. $C$, Relation between correct responses and reaction time for dark and light targets (normalized by the maximum percentage correct responses with the fellow eye, time bins: 0.05 s). Notice the logarithmic scale of the $x$-axis. $D$, Average accuracy (left) and reaction time (right) for darks (D) and lights (L). ${ }^{* *} p<0.001$. Error bars indicate \pm SEM.

the light targets at all (Fig. 3E, observer A.H.; chance level: 33\% for three target variations). In the absence of vision for light targets, observers with severe amblyopia could spend a long time looking for the targets in some trials or just give up and quickly hit a random key in other trials. Paradoxically, observers with severe amblyopia appeared to know when light targets were present in the screen but did not know the number because the targets blended in the background noise. The dark-light accuracy differences be- tween fellow and amblyopic eyes were significant in each participant with a visual acuity lower than $0.35 \log$ MAR in the amblyopic eye (Fig. $3 D-F$ ), and in the average across all amblyopic observers (Fig. $3 G-I ; n=15)$. Therefore, we conclude that amblyopia increases the dominance of dark stimuli in visual salience.

Our results also revealed a strong correlation between dark dominance and amblyopia severity (measured as a $\log$ MAR difference in visual acuity between amblyopic and fellow eyes). As 

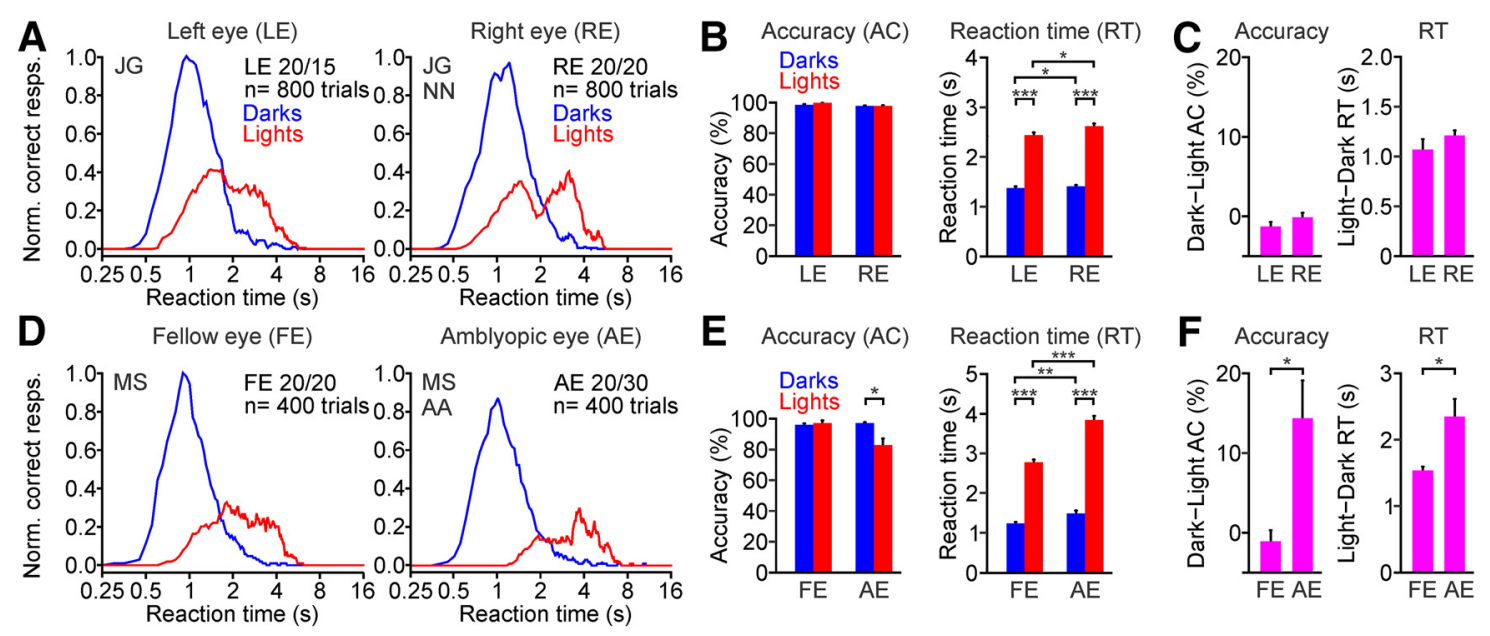

E Accuracy (AC)

Reaction time (RT)
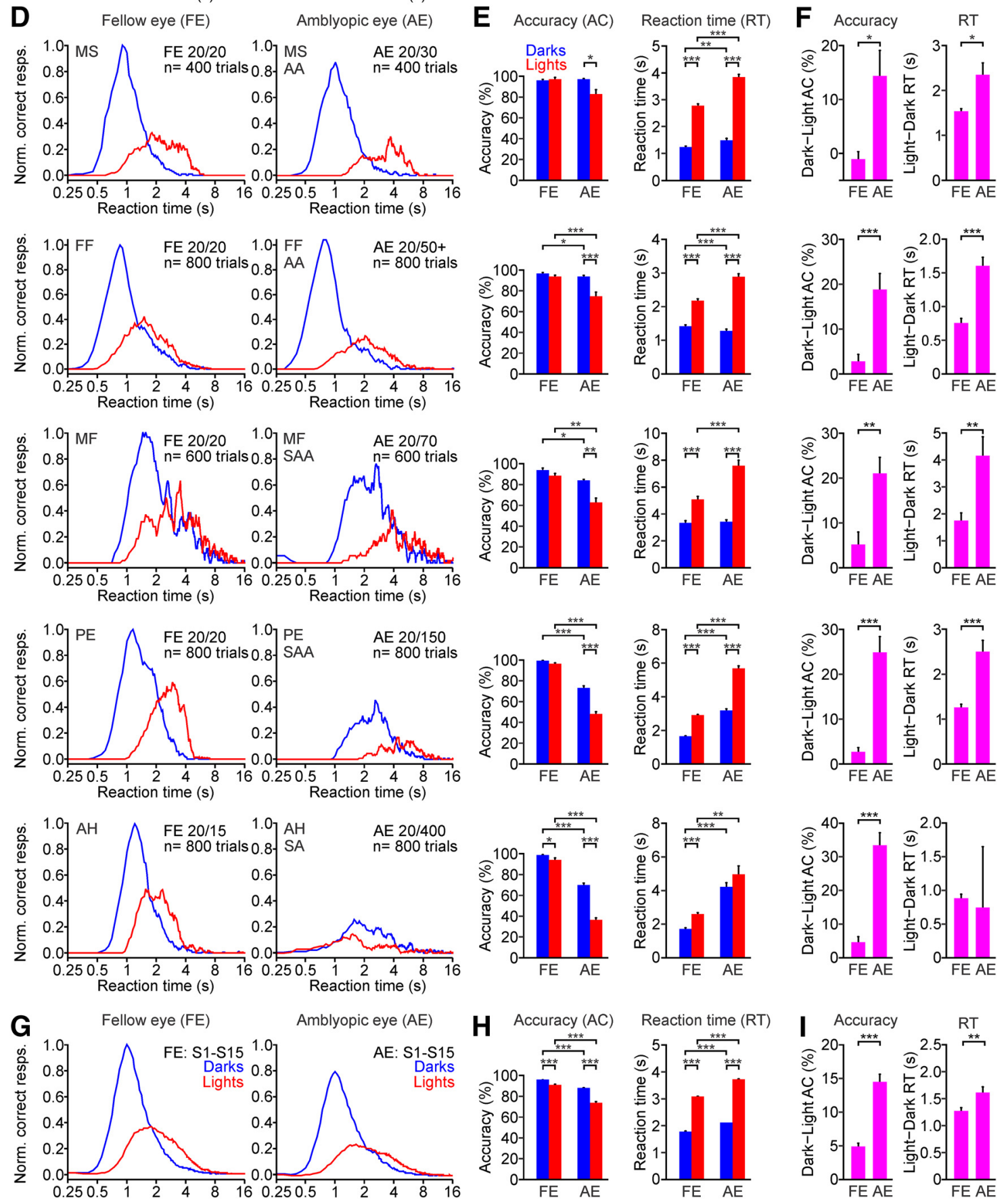

Figure 3. Amblyopia affects more the visual salience of lights than darks. A, Relation between correct responses and reaction time for dark and light targets measured with the left eye (LE; left) and right eye (RE; right) of a near-normal (NN) observer. The observer is identified by two capital letters (J.G.). The best-corrected visual acuity and the number of trials ( $n$ ) for each eye are shown next to the histogram. $\boldsymbol{B}$, Average accuracy (left) and reaction time (right) for responses to dark and light stimuli measured with the LE and RE of one example near-normal observer. $\boldsymbol{C}$, Average differences in accuracy (left) and reaction time (RT; right) between darks and lights measured in the left and right eyes of one example near-normal observer. $\boldsymbol{D}-\boldsymbol{F}$, Same as $\boldsymbol{A}-\boldsymbol{C}$ but for the fellow eye (FE; left) and amblyopic eye (AE; right) of five amblyopic observers ordered by the magnitude of light-dark asymmetries, from lowest (top) to highest (bottom). Every row corresponds to a different observer identified by two capital letters (e.g., M.S. for observer at the top). The type of amblyopia of each observer is shown next to the amblyopic eye histogram and is identified as AA, SA, and SAA. G-I, Same as $\boldsymbol{A}-\mathbf{C}$ but for the average of 15 amblyopic observers (S1-S15). ${ }^{*} p<0.05,{ }^{* *} p<0.01,{ }^{* * *} p<0.001$. Error bars indicate \pm SEM. 

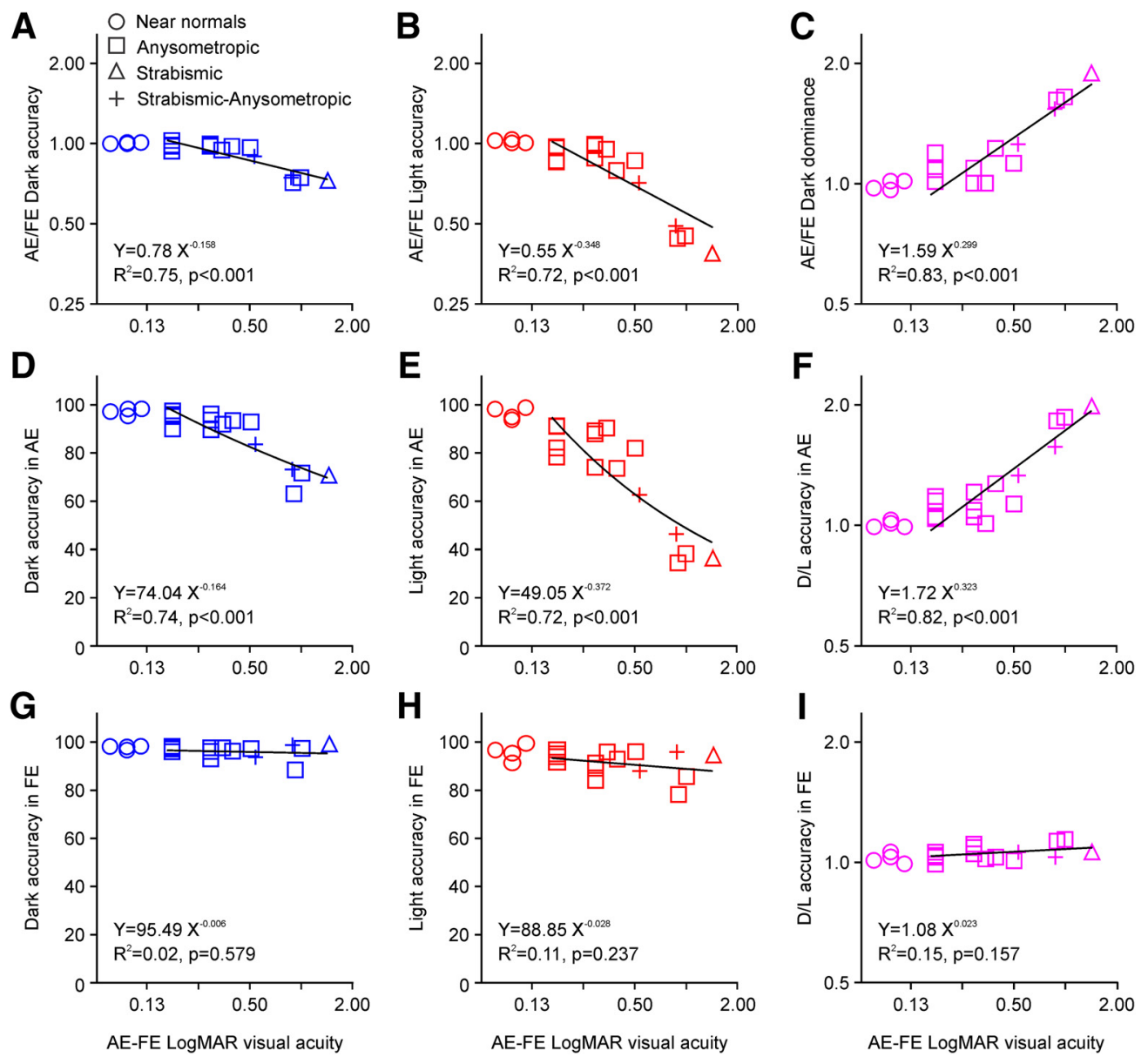

Figure 4. Dark dominance of visual salience is correlated with the severity of amblyopia. $\boldsymbol{A}$, Ratio between amblyopic and fellow eyes for dark-target accuracy (blue) is correlated with severity of amblyopia [estimated from the difference in best-corrected visual acuity between amblyopic (AE) and fellow eye (FE)]. The plot shows the data from near normal observers (circles; AE: eye with lowest visual acuity), AA (squares), SA (triangle), and SAA (crosses). The line shows a power regression function fit to the data for amblyopes (equations, goodness of fit and significance shown at the bottom). $\boldsymbol{B}$, Same as $\boldsymbol{A}$ for light-target accuracy (red). C, Same as $\boldsymbol{A}$ for dark dominance (ratio between dark accuracy and light accuracy). $\boldsymbol{D}$, Same as $\boldsymbol{A}$ for dark-target accuracy with the amblyopic eye. $\boldsymbol{E}$, Same as $\boldsymbol{A}$ for light-target accuracy with the amblyopic eye. $\boldsymbol{F}$, Same as $\boldsymbol{A}$ for dark dominance with the amblyopic eye. $\mathbf{G}-\boldsymbol{I}$, Same as $\boldsymbol{D}-\boldsymbol{F}$ for the FE.

amblyopia severity increased across observers, task performance decreased less for dark (Fig. 4A) than light targets (Fig. 4B) making visual salience more dark dominated (Fig. 4C; dark dominance: accuracy for darks/accuracy for lights). Amblyopia severity was strongly correlated with the ratio between amblyopic and fellow eyes for dark accuracy (Fig. $4 A ; R^{2}=0.75, p<0.001$, $n=15$ ), light accuracy (Fig. $4 B ; R^{2}=0.72, p<0.001, n=15$ ), and dark dominance (Fig. $4 C ; R^{2}=0.83, p<0.001, n=15$ ). The decrease in the amblyopic/fellow accuracy ratio for light targets was strongest in the four observers with the largest logMAR difference in visual acuity but the correlation was still significant if these four observers were excluded (Fig. $4 C ; R^{2}=0.54, p=0.010$, $n=11)$. Amblyopia severity was strongly correlated with the accuracy of the amblyopic eye for dark targets (Fig. $4 D ; R^{2}=$ 0.74, $p<0.001, n=15$ ), light targets (Fig. $4 E ; R^{2}=0.72, p<$ $0.001, n=15$ ), and with the ratio of dark/light accuracy (Fig. $4 F$; $\left.R^{2}=0.82, p<0.001, n=15\right)$. In contrast, there was no correlation between amblyopia severity and any measure of accuracy for the fellow eye (Fig. $4 G-I$ ). These results strongly suggest that amblyopia decreases more the visual salience of light than dark targets, as expected if amblyopia affected $\mathrm{ON}$ visual cortical pathways more than OFF.

Interestingly, the dark-light differences in visual salience not only increased in anisometropic amblyopes but also in strabismic amblyopes. In fact, the largest dark-light differences that we report were measured in a strabismic amblyope that had the most severe visual acuity loss among all observers selected for the data analysis (Figs. 4, right, triangles, A.H.: 20/400 in amblyopic eye, and $3 D-F$, bottom). This strabismic amblyope had a relatively small anisometropia $(+1.25$ in amblyopic eye vs -0.25 in the fellow eye) and, therefore, the large dark-light asymmetry was unlikely to be caused by optical blur. We hypothesize that darklight asymmetries originate in strabismic amblyopia through a mechanism that reduces cortical responses to high spatial frequencies, which may not be optical blur. Consistent with this hypothesis, strabismic monkeys that did not experience optical blur as infants show a pronounced reduction in cortical responses to high spatial frequencies (Kiorpes et al., 1998). It is possible that the strong interocular cortical suppression caused by diplopia affects cortical responses more to high than low spatial frequencies (Kwon et al., 2015) because the infant cortical spatial frequency tuning is low pass (Banks and Salapatek, 1978). Under this framework, anisometropic and strabismic amblyopia could both increase dark-light visual asymmetries through a reduction in cortical responses to high spatial frequencies. In anisometric amblyopia, optical blur would remove the high spatial frequencies already in the eye. In strabismic amblyopia, intero- 

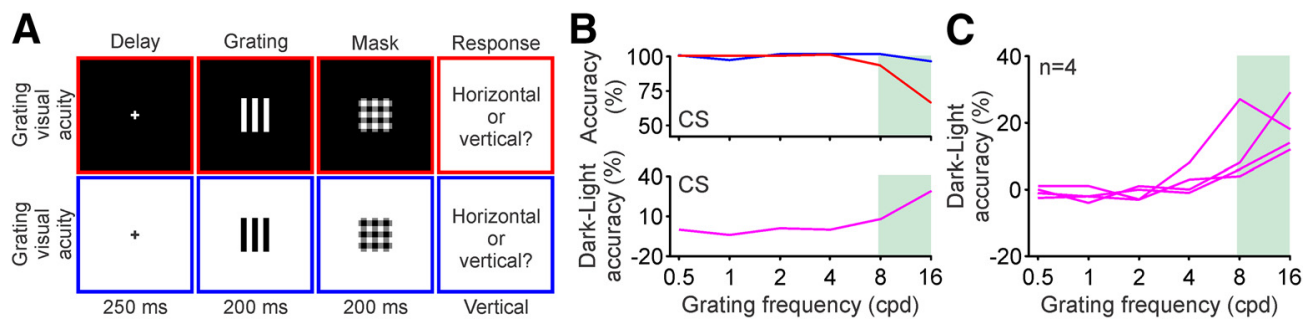

Figure 5. Grating visual acuity for darks and lights measured in control subjects. $A$, Grating visual acuity was measured with half-rectified gratings followed by a mask. Both gratings and mask lasted $200 \mathrm{~ms}$ and were presented after a $250 \mathrm{~ms}$ delay. Gratings could be either light $\left(312 \mathrm{~cd} / \mathrm{m}^{2}\right)$ on a dark $\left(0.5 \mathrm{~cd} / \mathrm{m}^{2}\right)$, were presented in dark background $\left(0.5 \mathrm{~cd} / \mathrm{m}^{2}\right.$, top) or light background $\left(312 \mathrm{~cd} / \mathrm{m}^{2}\right.$, bottom) and varied in spatial frequency $(0.5-16 \mathrm{cpd})$. Observers had to report the orientation of the grating. $\boldsymbol{B}$, Top, Measurements of visual acuity for light (red) and dark (blue) gratings in a control subject (CS). Bottom, The differences in accuracy between dark and light gratings. The green shading highlights the high spatial frequencies at which dark-light differences in visual acuity are observed. C, Dark-light accuracy functions for four control subjects.

cular suppression would reduce responses to high spatial frequencies in the cortex.

\section{Amblyopia affects grating visual acuity of light stimuli more than dark stimuli}

Our hypothesis also predicts that amblyopia should affect visual acuity more when measured with light than dark gratings. OFF cortical responses to dark stimuli increase roughly linearly with luminance contrast. However, ON cortical responses to light stimuli saturate at low luminance contrasts. We call this ON luminance/response saturation neuronal blur because it blurs light stimuli through neuronal response saturation instead of optics (Kremkow et al., 2014; Pons et al., 2017; Jansen et al., 2019). Unlike optical blur, the neuronal blur expands more the retinal representation of light than dark stimuli, making narrow gaps between closely-spaced bars harder to see when the bars are light. Therefore, we predicted that the neuronal blur should reduce visual acuity more in amblyopia when measured with light than dark gratings.

We measured grating visual acuity in amblyopic observers with the same task that we used previously in normal observers (Pons et al., 2017). The stimuli were half-wave rectified gratings that could be light $\left(312 \mathrm{~cd} / \mathrm{m}^{2}\right)$ or dark $\left(0.5 \mathrm{~cd} / \mathrm{m}^{2}\right)$, and that were presented in dark $\left(0.5 \mathrm{~cd} / \mathrm{m}^{2}\right)$ or light $\left(312 \mathrm{~cd} / \mathrm{m}^{2}\right)$ backgrounds at different spatial frequencies $(0.5-16 \mathrm{cpd})$. The observers had to report the orientation of a grating presented monocularly for 200 ms, followed by a mask (Fig. 5A).

The neuronal blur causes an expansion of light stimuli that is large enough to fill in the narrow gaps of high-frequency gratings but not the broader gaps of gratings with lower frequency. Consequently, the dark-light differences in grating visual acuity appeared only at the highest grating frequencies visible in both normal observers (Fig. $5 B, C$ ) and near-normal observers (Fig. $6 A$, top, observer J.G.). Moreover, in observers with moderate amblyopia, the dark-light differences in visual acuity shifted to lower spatial frequencies (i.e., the highest grating frequencies that the amblyopic eye could see) and became more pronounced in the amblyopic eye than the fellow eye (Fig. 6A, observers between J.G. and A.H.). Finally, as we predicted, because observers with severe amblyopia could not see the high grating frequencies, the dark-light differences in grating visual acuity became smaller for the amblyopic than the fellow eye (Fig. 6 A, bottom, observer A.H.; notice that A.H. failed to see grating frequencies $\left.>1 \mathrm{cycle} /{ }^{\circ}\right)$. The lack of dark-light differences in observers with severe amblyopia is exactly what would be expected from the local effect of neuronal blur. Just as for normal observers tested under low light (Pons et al., 2017), dark-light differences in grating visual acuity are greatly reduced in observers that cannot discriminate grating high frequencies.

On average, dark-light differences across all amblyopic observers were most pronounced at $16 \mathrm{cpd}$ when using the fellow eye (Fig. $6 B$ ) and at 4 cpd when using the amblyopic eye (Fig. 6C). When measured at the grating frequency cutoff for the amblyopic eye (i.e., the grating frequency that made amblyopic-eye accuracy for dark stimuli drop to $<90 \%$ ), the dark-light differences in grating visual acuity were five times larger for the amblyopic than the fellow eye in observers with moderate amblyopia (fellow eye: $2.25 \pm 1.29 \%$, amblyopic eye: $11.62 \pm 1.90 \%, n=12, p=0.001$, Wilcoxon test). If observers with more severe amblyopia were also included, the dark-light differences for the amblyopic eye were reduced but remained significantly larger than for the fellow eye (Fig. $6 D, E$; fellow eye: $2.04 \pm 1.11 \%$, amblyopic eye: $9.15 \pm$ $2.33 \%, n=14, p=0.007$, Wilcoxon test). Across all amblyopic observers, the magnitude of dark-light differences in grating visual acuity were negatively correlated with amblyopia severity (Fig. 6F; $r=-0.78, p=0.001, n=14$ ), as predicted from the effects of neuronal blur (Kremkow et al., 2014; Pons et al., 2017). Because dark-light differences in grating visual acuity could only be measured within a narrow range of grating frequencies (i.e., the highest frequencies visible) and amblyopia severity (Fig. 6A), we could not demonstrate an increase of dark dominance in grating acuity with amblyopia progression.

Dark-light differences in vision were smaller when tested with the fellow eyes of amblyopic observers than the eyes of control observers. However, the difference did not reach significance when measured at both $4-8 \mathrm{cpd}(4.19 \pm 1.27 \%$ vs $6.87 \pm 3.11 \%$, $n=12$ fellow and 4 control eyes, $p=0.49$, Wilcoxon test) and 16 cpd ( $8.17 \pm 2.88 \%$ vs $18.25 \pm 3.79 \%, n=12$ fellow and 4 control eyes, $p=0.16$, Wilcoxon test, $200 \mathrm{~ms}$ presentations for both groups).

\section{Amblyopia affects ON visual pathways more than OFF}

A computational model that incorporates neuronal blur in visual processing can stimulate the development of amblyopia, the psychophysical dark dominance that we demonstrate and other amblyopia deficits in cortical suppression (Shooner et al., 2017). The model also provides a mechanism to explain how optical blur and strabismus can affect $\mathrm{ON}$ visual cortical pathways more than OFF during cortical development.

The model has four main stages: input, retinal, thalamocortical, and cortical stage (Fig. 7A-D; see Materials and Methods). The input stage simulates the optics of the eye and uses a Gaussian function to blur images of dark and light stimuli (Fig. 7A). The retinal stage simulates the neuronal processing from $\mathrm{ON}$ and $\mathrm{OFF}$ 


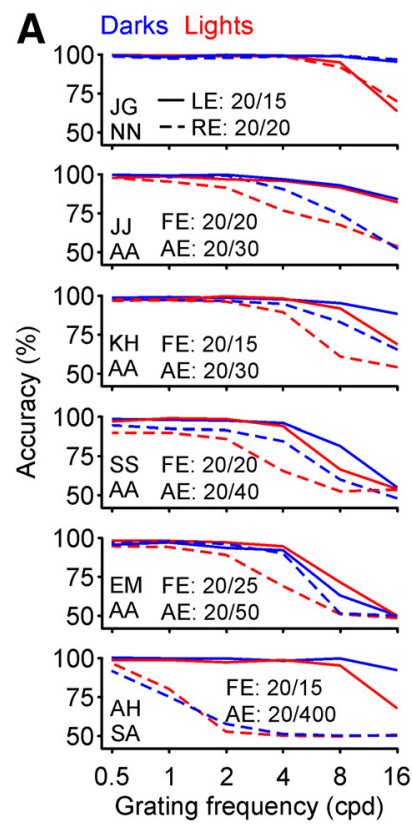

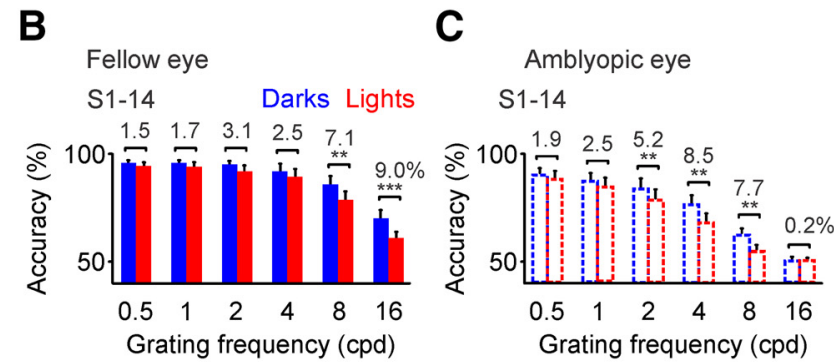

D

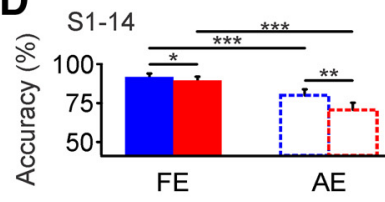

E $\quad$ S1-14

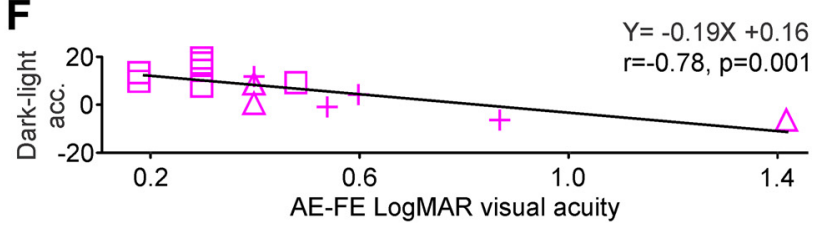

Figure 6. Amblyopia reduces visual acuity more for lights than darks. $A$, Top, Measurements of visual acuity for light (red) and dark (blue) gratings shown as a function of spatial frequency ( $x$-axis). Visual acuity was measured in the left (LE; continuous lines) and right eye ( $\mathrm{RE}$; dotted lines) of one near-normal (NN) observer (J.G.). The best-corrected visual acuities are shown for left and right eyes on the left. Bottom panels, below J.G., are the same as the top, but for the fellow eye (FE; continuous lines) and amblyopic eye (AE; dotted lines) of five amblyopic observers. The type of amblyopia of each observer is shown on the left (4 examples of AA and 1 of $S A$ ). B. Average grating visual acuity measured for light (red) and dark (blue) gratings at six different spatial frequencies (0.5-16 cpd) in the fellow eye of al 14 amblyopic observers (S1-S14). The dark-light accuracy difference is shown on top of each spatial frequency bin. C, Same as $\boldsymbol{B}$ for the amblyopic eye of the 14 amblyopic observers. D, Average accuracy measured at the grating frequency cutoff for the amblyopic eye (the grating frequency that caused a drop $>90 \%$ in accuracy for dark targets seen through the amblyopic eye). Accuracy measurements are shown for the FEs and AEs of the 14 amblyopic observers. $E$, Average dark-light differences in accuracy for the same 14 observers. $F$, Dark-light differences in accuracy for grating visual acuity are negatively correlated with amblyopia severity in the 14 amblyopic observers. ${ }^{*} p<0.05,{ }^{* *} p<0.01,{ }^{* *} p<0.001$. Error bars indicate \pm SEM.

bipolar cells and applies greater neuronal blur to light than dark stimuli (Fig. 7B). The thalamocortical stage simulates the centersurround filtering of bipolar, retinal ganglion cells and thalamic cells through a convolution with a difference-of-Gaussians function (Fig. 7C). Finally, the cortical stage simulates the binocular integration of $\mathrm{ON}$ and $\mathrm{OFF}$ thalamocortical inputs through a weighted sum of the thalamocortical pathways and interocular suppression (Fig. 7D).

Our model reproduces our psychophysical results for visual salience (Fig. $7 E$ ), grating visual acuity (Fig. $7 F$ ), and the deficits in interocular suppression (Fig. 7G) demonstrated in the macaque amblyopic cortex (Shooner et al., 2017). The key component of the model is the neuronal blur, which is larger for ON than OFF visual pathways (Fig. $7 B$ ) and is amplified by optical blur (or any other mechanism that reduces cortical responses to high spatial frequencies). In the presence of defocus, the larger optical blur leads to a larger spatial distortion of light stimuli at the retinal stage (Fig. 7B). Conversely, in the complete absence of optical blur (which is not biologically realistic), neuronal responses at the retinal stage (Fig. $7 B$ ) should be identical to the input images (Fig. 7A, left).

In the presence of optical blur, neuronal blur makes ON responses weaker than OFF responses during cortical development because it reduces the value of the convolution at the thalamocortical stage for lights more than darks (Fig. 7C). The reduction of the convolution value simulates a decrease in the stimulus contrast driving $\mathrm{ON}$ visual responses. As $\mathrm{ON}$ visual responses become weaker, the ON cortical pathway from the amblyopic eye also becomes weaker. Conversely, because the optical blur is limited in the fellow eye, the synaptic weights of OFF and ON pathways driven by the fellow eye remain more similar during cortical development. In the adult, the ON pathway remains weaker than the OFF pathway and this difference is enough to reproduce the psychophysical dark dominance for both visual salience and grating visual acuity that we demonstrate (Fig. $7 E, F)$. The model can also reproduce deficits in cortical suppression (Fig. $7 G$ ) if we include a reduction in ON-luminance background suppression (Xing et al., 2014), as it would be expected from a weak ON cortical pathway.

Our developmental model can also reproduce the results for different types of amblyopia if we assume that both optical blur and strabismus reduce cortical responses to high spatial frequencies. The developmental model reduces the strength of ON cortical responses more than OFF as optical blur increases (Fig. $7 \mathrm{H}$ ) because optical blur makes the stimulus more dominated by lower spatial frequencies and low spatial frequencies drive weaker ON than OFF cortical responses (Kremkow et al., 2014; Jansen et al., 2019). The same result can be obtained if optical blur is kept constant at normal levels but there is another mechanism that reduces cortical responses to high spatial frequencies. For example, as cortical spatial resolution decreases, the OFF-ON differences in cortical responses increase (Fig. 7I). An interesting possibility is that diplopia enhances the suppression of cortical responses driven by the amblyopic eye across all stimulus spatial frequencies. However, because the cortex of human infants respond much stronger to stimuli of low than high spatial frequencies (Banks and Salapatek, 1978), the global response reduction completely eliminates cortical responses to high but not low spatial frequencies. Under this framework, any mechanism that reduces cortical responses to high spatial frequencies during development should increase the dark dominance of visual perception (Fig. 8). This mechanism could be optical blur in anisometropic amblyopia or cortical suppression in strabismic amblyopia. 


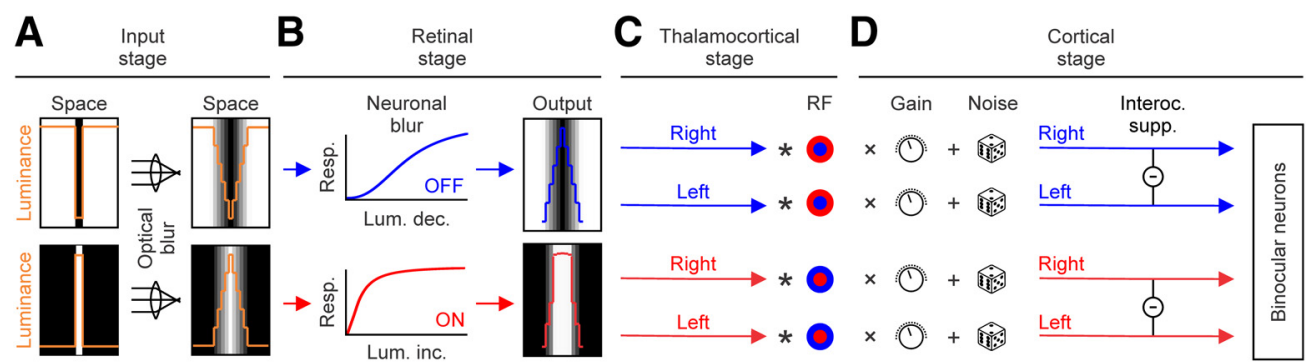

E Visual salience (data)

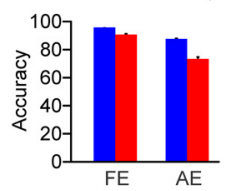

Visual salience (model)

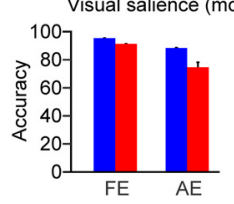

H

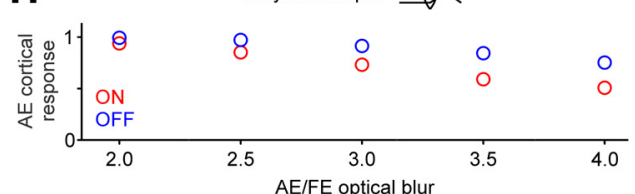

F Grating visual acuity (data)
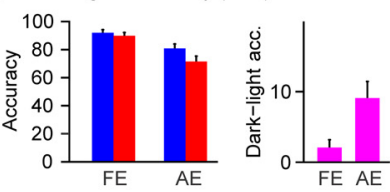

Grating visual acuity (model)

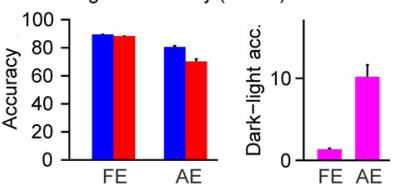

I
G

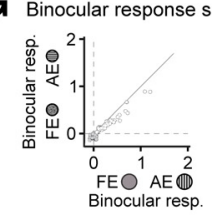

Binocular response suppression (model)

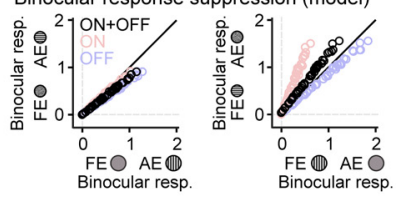

Strabismus 1

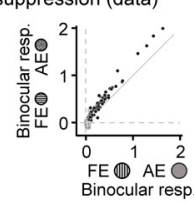

$\therefore$

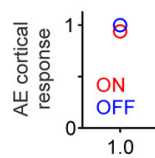

Figure 7. A computational model with neuronal blur can reproduce the amblyopia deficits. $\boldsymbol{A}-\boldsymbol{D}$, A model that incorporates a larger neuronal blur in the $0 \mathrm{~N}$ than the $0 \mathrm{FF}$ pathway accurately replicates the amblyopia deficits reported in our study. The neuronal blur reduces cortical responses more for light than dark stimuli, an effect that is amplified by optical blur or any other mechanism that reduces cortical responses to high spatial frequencies. During development, the response reduction leads to weaker ON than $0 \mathrm{FF}$ cortical pathways in the amblyopic eye. Resp.: response; Lum. inc.: luminance increment; Lum. dec.: luminance decrement; Interoc. supp.: interocular suppression. $\boldsymbol{E}$, Comparison of data (top) and model simulations (bottom) for salience task. Accuracy measurements are shown separately for light (red) and dark (blue) stimuli presented to the fellow (FE) and amblyopic eye (AE). Right, The dark-light differences (pink). $\boldsymbol{F}$, Same as $\boldsymbol{E}$ but for the visual acuity task. G, Model simulations for deficits in cortical response suppression reported by Shooner et al. (2017). Data from Shooner et al. (2017) are shown at the top (data points in gray are purely monocular sites) and response simulations at the bottom. $\boldsymbol{H}$, Simulations with developmental model illustrating how an increase in optical blur enhances $0 \mathrm{FF}-0 \mathrm{~N}$ differences in cortical responses. $\boldsymbol{I}$, Same as $\boldsymbol{H}$ but in simulations that kept the optical blur constant at normal levels while reducing the cortical spatial resolution in the amblyopic eye.

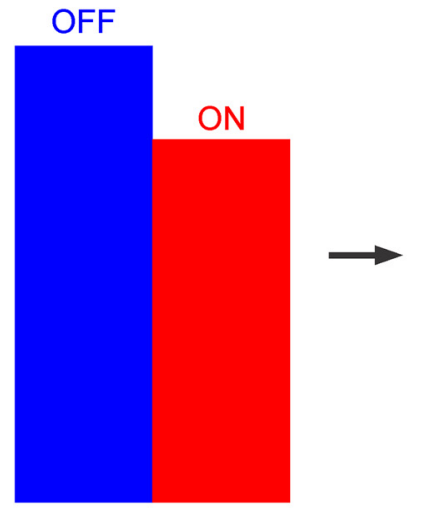

ON/OFF balance

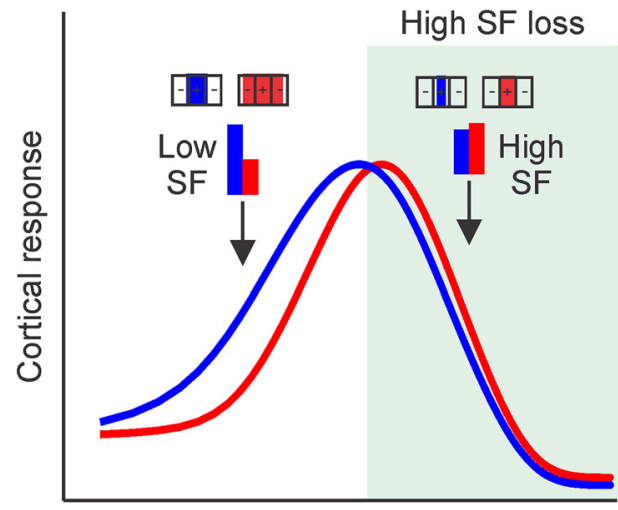

Spatial frequency (SF)

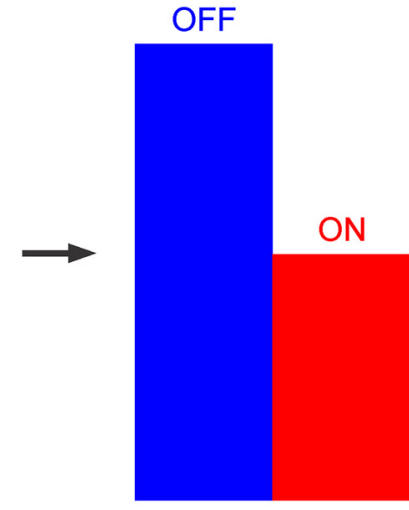

ON/OFF balance

Figure 8. Reduction of cortical responses to high spatial frequencies weakens more $\mathrm{ON}$ than $\mathrm{OFF}$ cortical pathways during brain development. Left, $0 \mathrm{FF}$ cortical responses (blue) are slightly stronger than $\mathrm{ON}$ cortical responses (red) in normal observers. Middle, $\mathrm{OFF}$ cortical responses are stronger than $\mathrm{ON}$ cortical responses at low spatial frequencies. Any process that reduces cortical responses to high spatial frequencies during cortical development should make cortical responses more 0FF dominated (green, response loss). Receptive fields at the top illustrate the expansion of light stimuli (red) relative to dark stimuli (blue) by neuronal blur (expansion by 2 times for illustration purposes). At high spatial frequencies, the expansion of light stimuli increases spatial summation within the positive region of the receptive field, making ON responses slightly stronger than OFF responses (although the expansion reduces grating visual acuity). At low spatial frequencies, the expansion of light stimuli increases spatial suppression within the negative region of the receptive field, making $0 \mathrm{~N}$ responses weaker than OFF responses. Right, Loss of cortical responses to high spatial frequencies affect more ON than OFF cortical responses (shorter red bars at right compared with left). 


\section{Discussion}

Our results demonstrate that amblyopia affects visual processing of light more than dark stimuli, making vision more dark dominated. We show that this dark dominance is strongly correlated with amblyopia severity and can be explained by a neuronal amplification of optical blur (or any other mechanism that reduces cortical responses to high spatial frequencies) during cortical development. Based on these results, we conclude that ON cortical pathways are more affected than OFF cortical pathways by visual disorders associated with a loss of cortical responses to high spatial frequencies.

\section{Visual deficits in amblyopia}

Amblyopia causes multiple visual deficits including a reduction in contrast sensitivity, spatial resolution, position acuity, and a disruption of form and motion integration (Kozma and Kiorpes, 2003; McKee et al., 2003; Kiorpes et al., 2006; Whitney and Levi, 2011). These sensory deficits are likely to originate in primary visual cortex and be further amplified at later cortical stages (Wiesel and Hubel, 1963; Hubel et al., 1977; Kiorpes, 2006; Levi, 2006; Clavagnier et al., 2015). It is commonly assumed that amblyopia affects similarly ON and OFF cortical responses. This assumption is probably based on the traditional notion that $\mathrm{ON}$ and OFF visual pathways segregate in retina and thalamus but combine in visual cortex (Hubel and Wiesel, 1962). Because amblyopia does not greatly affect retinal and thalamic function, there was no reason to believe that $\mathrm{ON}$ and $\mathrm{OFF}$ visual pathways should be differently affected. However, there is increasingly stronger evidence that $\mathrm{ON}$ and OFF pathways remain segregated in visual cortex as is also the case for the pathways from the two eyes (Kremkow and Alonso, 2018). The four pathways (contralateral eye, ipsilateral eye, ON, and OFF) combine in visual cortex to maximize the binocular processing of diverse lightdark patterns but they also remain segregated. Consequently, losing one eye does not compromise vision through the other eye and losing the ON pathway does not compromise vision through the OFF pathway. Without ON pathway, humans and monkeys can have normal visual acuity and perform most visual tasks except those that require seeing light targets on dark backgrounds and discriminating low-contrast patterns (Schiller et al., 1986; Dryja et al., 2005).

Just as the contralateral eye is better represented than the ipsilateral eye in visual cortex (Wiesel and Hubel, 1963; Adams et al., 2007), the OFF pathway is better represented than the ON pathway (Jin et al., 2008; Yeh et al., 2009). The OFF pathway also has higher retinotopic precision (Kremkow et al., 2016; Lee et al., 2016) and generates responses that are stronger, faster, and more linearly related with luminance contrast than the ON pathway (Zemon et al., 1988; Jin et al., 2008, 2011a,b; Yeh et al., 2009; Kremkow et al., 2014). The luminance/response saturation (that we call neuronal blur) is also more pronounced in ON than OFF pathways, is amplified by optical blur and distorts more the size of light than dark stimuli (Kremkow et al., 2014; Pons et al., 2017). Therefore, we predicted that optical blur should affect ON pathways more than OFF during cortical development. Consistent with this prediction, amblyopic observers that experienced sustained optical blur early in life (or lost cortical responses to high spatial frequencies through other mechanisms) had more pronounced visual deficits when tested with light than dark stimuli. These results highlight the importance of measuring amblyopia deficits separately for lights and darks, in addition to measuring the combined ON-OFF deficits with gratings or checkerboards.
Amblyopia can also affect the fellow eye; however, deficits in the fellow eye were subtle in our measurements. If anything, the dark-light differences were smaller for the fellow eyes of amblyopic observers than the eyes of control observers. In visual salience, the average dark-light difference was only $5 \%$ for fellow eyes, whereas it ranged between 8 and $11 \%$ for control eyes measured in our previous studies [9\% in Komban et al. (2011), 8\% in Zhao et al. (2015), 11\% in Pons et al. (2017)]. In grating visual acuity, the dark-light differences were also lower for fellow than control eyes ( 8.83 vs $18.25 \%$ for $16 \mathrm{cpd} / 200 \mathrm{~ms}$ gratings) but the difference did not reach significance. Therefore, whereas there is a tendency for fellow eyes to show less dark-light differences than the average control eye, the variability across subjects complicates the interpretation of this trend.

\section{Neuronal mechanisms of amblyopia}

Our results suggest that optical blur changes the balance between $\mathrm{ON}$ and OFF cortical responses just as monocular deprivation changes the balance between contralateral- and ipsilateral-eye responses. In turn, they suggest that sustained exposure to optical blur weakens the ON pathway during cortical development just as monocular deprivation weakens vision through the deprived eye. Consistent with this hypothesis, a computational model that incorporates $\mathrm{ON}-\mathrm{OFF}$ differences in neuronal blur reproduces the different visual deficits for ON than OFF cortical pathways that we demonstrate in amblyopia (Kremkow et al., 2014; Pons et al., 2017). The model can also reproduce the results for strabismic amblyopia if we reduce the cortical spatial resolution of the amblyopic eye while keeping optical blur at normal levels.

In our model, ON cortical responses saturate more with luminance contrast than OFF cortical responses and this saturation causes an expansion of light image features represented in the retina (Kremkow et al., 2014; Pons et al., 2017). In turn, the size expansion leads to weaker ON than OFF signals at the thalamocortical stage and weaker ON than OFF cortical responses. Our model weakens cortical responses from the amblyopic eye both because the cortical input becomes weaker and the interocular suppression from the fellow eye stronger. The model also increases the baseline noise of cortical responses driven by the amblyopic eye because the ON background luminance suppression becomes weaker (Xing et al., 2014).

There are several striking resemblances between the visual deficits caused by amblyopia and those caused by loss of ONpathway function that deserved to be discussed. In humans, macaques and mice, the loss of $\mathrm{ON}$ visual function causes a pronounced reduction of contrast sensitivity for light-dark stimulus patterns (Schiller et al., 1986; Dryja et al., 2005; Sarnaik et al., 2014), which is one of the first visual deficits described in amblyopia (Levi and Harwerth, 1977). This sensitivity loss is expected from the higher contrast sensitivity and contrast/response gain of ON cortical pathways when compared with OFF cortical pathways (Kremkow et al., 2014; Pons et al., 2017). Because the loss of $\mathrm{ON}$ visual function makes cortical luminance/response functions more OFF dominated, amblyopia should also increase the contrast that generates $50 \%$ of the maximum response $\left(\mathrm{C}_{50}\right)$ and reduce the contrast/response saturation. In addition, because the reduction in $\mathrm{ON}$ background luminance suppression increases cortical noise, the loss of $\mathrm{ON}$ visual function should also increase cortical noise. Therefore, a loss of $\mathrm{ON}$ visual function should cause correlated changes in three cortical parameters: $\mathrm{C}_{50}$, contrast/response saturation and noise. These correlated changes were recently demonstrated in the cortex of amblyopic macaques (Wang et al., 2017). 


\section{Future amblyopia treatments}

Hippocrates treated amblyopia with a healthy diet and physical exercise, and it was not until the 1700s that physicians started covering the fellow eye to "strengthen" vision through the amblyopic eye. Over the years, the trust in this old amblyopia treatment became so strong that some doctors in the 1900s proposed improving compliance by suturing the eyelids of fellow eyes in children (Loudon and Simonsz, 2005). In our days, clinicians still treat amblyopia by covering the fellow eye while correcting the refractive error of the amblyopic eye. However, whereas covering the fellow eye has been used for hundreds of years, its success is limited and has a high risk of recurrence. Promising new approaches use a more efficient stimulation of the amblyopic eye with difficult tasks or videogames while still covering the fellow eye or reducing its contrast (Polat et al., 2004; Li et al., 2013; Gambacorta et al., 2018). Other more experimental approaches in animals boost recovery through retinal inactivation (Fong et al., 2016) or by reducing binocular activity through exposure to darkness (He et al., 2007). All these approaches are promising but still fall short of providing full amblyopia recovery in humans.

Our results may open new paths for amblyopia treatments. Recent studies demonstrate that the cortical segregation of $\mathrm{ON}$ and OFF pathways may be as pronounced as the segregation for contralateral/ipsilateral eyes or magnocellular/parvocellular pathways (Jin et al., 2011a; Kremkow et al., 2016; Lee et al., 2016). Recent work is also revealing pronounced functional differences between ON and OFF cortical pathways, with low spatial frequencies, optical blur and low light making vision more OFF dominated (Onat et al., 2011; Kremkow et al., 2014; Pons et al., 2017; Jansen et al., 2019) and slow motion making vision and cortical responses more ON dominated (Luo-Li et al., 2018; Mazade et al., 2019). Therefore, our results may inspire a new generation of amblyopia treatments that can take advantage of the different $\mathrm{ON}$ and OFF stimulus preferences to selectively strengthen a weak ON cortical pathway.

\section{References}

Adams DL, Sincich LC, Horton JC (2007) Complete pattern of ocular dominance columns in human primary visual cortex. J Neurosci 27:1039110403.

Banks MS, Salapatek P (1978) Acuity and contrast sensitivity in 1-, 2-, and 3-month-old human infants. Invest Ophthalmol Vis Sci 17:361-365.

Brainard DH (1997) The psychophysics toolbox. Spat Vis 10:433-436.

Campbell FW, Gubisch RW (1966) Optical quality of the human eye. J Physiol 186:558-578.

Chichilnisky EJ, Kalmar RS (2002) Functional asymmetries in ON and OFF ganglion cells of primate retina. J Neurosci 22:2737-2747.

Chubb C, Nam JH (2000) Variance of high contrast textures is sensed using negative half-wave rectification. Vision Res 40:1677-1694.

Chubb C, Landy MS, Econopouly J (2004) A visual mechanism tuned to black. Vision Res 44:3223-3232.

Clavagnier S, Dumoulin SO, Hess RF (2015) Is the cortical deficit in amblyopia due to reduced cortical magnification, loss of neural resolution, or neural disorganization? J Neurosci 35:14740-14755.

Cooper EA, Norcia AM (2015) Predicting cortical dark/bright asymmetries from natural image statistics and early visual transforms. PLoS Comput Biol 11:e1004268.

Dacey DM, Petersen MR (1992) Dendritic field size and morphology of midget and parasol ganglion cells of the human retina. Proc Natl Acad Sci U S A 89:9666-9670.

Ding J, Levi DM (2014) Rebalancing binocular vision in amblyopia. Ophthalmic Physiol Opt 34:199-213.

Ding J, Sperling G (2006) A gain-control theory of binocular combination. Proc Natl Acad Sci U S A 103:1141-1146.

Ding J, Klein SA, Levi DM (2013) Binocular combination in abnormal binocular vision. J Vis 13(2):14 1-31.

Dryja TP, McGee TL, Berson EL, Fishman GA, Sandberg MA, Alexander KR,
Derlacki DJ, Rajagopalan AS (2005) Night blindness and abnormal cone electroretinogram ON responses in patients with mutations in the GRM6 gene encoding mGluR6. Proc Natl Acad Sci U S A 102:4884-4889.

Fong MF, Mitchell DE, Duffy KR, Bear MF (2016) Rapid recovery from the effects of early monocular deprivation is enabled by temporary inactivation of the retinas. Proc Natl Acad Sci U S A 113:14139-14144.

Gambacorta C, Nahum M, Vedamurthy I, Bayliss J, Jordan J, Bavelier D, Levi DM (2018) An action video game for the treatment of amblyopia in children: a feasibility study. Vision Res 148:1-14.

He HY, Ray B, Dennis K, Quinlan EM (2007) Experience-dependent recovery of vision following chronic deprivation amblyopia. Nat Neurosci 10:1134-1136.

Holmes JM, Clarke MP (2006) Amblyopia. Lancet 367:1343-1351.

Huang CB, Zhou J, Zhou Y, Lu ZL (2010) Contrast and phase combination in binocular vision. PLoS One 5:e15075.

Hubel DH, Wiesel TN (1962) Receptive fields, binocular interaction and functional architecture in the cat's visual cortex. J Physiol 160:106-154.

Hubel DH, Wiesel TN, LeVay S (1977) Plasticity of ocular dominance columns in monkey striate cortex. Philos Trans R Soc Lond B Biol Sci 278:377-409.

Jansen M, Jin J, Li X, Lashgari R, Kremkow J, Bereshpolova Y, Swadlow HA, Zaidi Q, Alonso JM (2019) Cortical balance between ON and OFF visual responses is modulated by the spatial properties of the visual stimulus. Cereb Cortex 29:336-355.

Jimenez LO, Tring E, Trachtenberg JT, Ringach DL (2018) Local tuning biases in mouse primary visual cortex. J Neurophysiol 120:274-280.

Jin JZ, Weng C, Yeh CI, Gordon JA, Ruthazer ES, Stryker MP, Swadlow HA, Alonso JM (2008) On and off domains of geniculate afferents in cat primary visual cortex. Nat Neurosci 11:88-94.

Jin J, Wang Y, Swadlow HA, Alonso JM (2011a) Population receptive fields of $\mathrm{ON}$ and $\mathrm{OFF}$ thalamic inputs to an orientation column in visual cortex. Nat Neurosci 14:232-238.

Jin J, Wang Y, Lashgari R, Swadlow HA, Alonso JM (2011b) Faster thalamocortical processing for dark than light visual targets. J Neurosci 31:1747117479.

Kiorpes L (2006) Visual processing in amblyopia: animal studies. Strabismus 14:3-10.

Kiorpes L, Kiper DC, O’Keefe LP, Cavanaugh JR, Movshon JA (1998) Neuronal correlates of amblyopia in the visual cortex of macaque monkeys with experimental strabismus and anisometropia. J Neurosci 18:64116424.

Kiorpes L, Tang C, Movshon JA (2006) Sensitivity to visual motion in amblyopic macaque monkeys. Vis Neurosci 23:247-256.

Komban SJ, Alonso JM, Zaidi Q (2011) Darks are processed faster than lights. J Neurosci 31:8654-8658.

Komban SJ, Kremkow J, Jin J, Wang Y, Lashgari R, Li X, Zaidi Q, Alonso JM (2014) Neuronal and perceptual differences in the temporal processing of darks and lights. Neuron 82:224-234.

Kozma P, Kiorpes L (2003) Contour integration in amblyopic monkeys. Vis Neurosci 20:577-588.

Kremkow J, Alonso JM (2018) Thalamocortical circuits and functional architecture. Annu Rev Vis Sci 4:263-285.

Kremkow J, Jin J, Komban SJ, Wang Y, Lashgari R, Li X, Jansen M, Zaidi Q, Alonso JM (2014) Neuronal nonlinearity explains greater visual spatial resolution for darks than lights. Proc Natl Acad Sci U S A 111:3170-3175.

Kremkow J, Jin J, Wang Y, Alonso JM (2016) Principles underlying sensory map topography in primary visual cortex. Nature 533:52-57.

Kwon M, Wiecek E, Dakin SC, Bex PJ (2015) Spatial-frequency dependent binocular imbalance in amblyopia. Sci Rep 5:17181.

Lee KS, Huang X, Fitzpatrick D (2016) Topology of ON and OFF inputs in visual cortex enables an invariant columnar architecture. Nature 533:90 94.

Levi DM (2006) Visual processing in amblyopia: human studies. Strabismus 14:11-19.

Levi DM, Harwerth RS (1977) Spatio-temporal interactions in anisometropic and strabismic amblyopia. Invest Ophthalmol Vis Sci 16:90-95.

Li J, Thompson B, Deng D, Chan LY, Yu M, Hess RF (2013) Dichoptic training enables the adult amblyopic brain to learn. Curr Biol 23:R308R309.

Loudon SE, Simonsz HJ (2005) The history of the treatment of amblyopia. Strabismus 13:93-106.

Luo-Li G, Mazade R, Zaidi Q, Alonso JM, Freeman AW (2018) Motion 
changes response balance between ON and OFF visual pathways. Commun Biol 1:60.

Mazade R, Jin J, Pons C, Alonso JM (2019) Functional specialization of ON and OFF cortical pathways for global-slow and local-fast vision. Cell Rep 27:2881-2894.e5.

McConnell SK, LeVay S (1984) Segregation of on- and off-center afferents in mink visual cortex. Proc Natl Acad Sci U S A 81:1590-1593.

McKee SP, Levi DM, Movshon JA (2003) The pattern of visual deficits in amblyopia. J Vis 3:380-405.

Norton TT, Rager G, Kretz R (1985) ON and OFF regions in layer IV of striate cortex. Brain Res 327:319-323.

Onat S, Nortmann N, Rekauzke S, König P, Jancke D (2011) Independent encoding of grating motion across stationary feature maps in primary visual cortex visualized with voltage-sensitive dye imaging. Neuroimage 55:1763-1770.

Polat U, Ma-Naim T, Belkin M, Sagi D (2004) Improving vision in adult amblyopia by perceptual learning. Proc Natl Acad Sci USA 101:6692-6697.

Pons C, Mazade R, Jin J, Dul MW, Zaidi Q, Alonso JM (2017) Neuronal mechanisms underlying differences in spatial resolution between darks and lights in human vision. J Vis 17(4):5 1-24.

Ratliff CP, Borghuis BG, Kao YH, Sterling P, Balasubramanian V (2010) Retina is structured to process an excess of darkness in natural scenes. Proc Natl Acad Sci U S A 107:17368-17373.

Rekauzke S, Nortmann N, Staadt R, Hock HS, Schöner G, Jancke D (2016) Temporal asymmetry in dark-bright processing initiates propagating activity across primary visual cortex. J Neurosci 36:1902-1913.

Sarnaik R, Chen H, Liu X, Cang J (2014) Genetic disruption of the on visual pathway affects cortical orientation selectivity and contrast sensitivity in mice. J Neurophysiol 111:2276-2286.

Schiller PH, Sandell JH, Maunsell JH (1986) Functions of the ON and OFF channels of the visual system. Nature 322:824-825.

Shooner C, Hallum LE, Kumbhani RD, García-Marín V, Kelly JG, Majaj NJ, Movshon JA, Kiorpes L (2017) Asymmetric dichoptic masking in visual cortex of amblyopic macaque monkeys. J Neurosci 37:8734-8741.
Smith GB, Whitney DE, Fitzpatrick D (2015) Modular representation of luminance polarity in the superficial layers of primary visual cortex. Neuron 88:805-818.

Wang Y, Jin J, Kremkow J, Lashgari R, Komban SJ, Alonso JM (2015) Columnar organization of spatial phase in visual cortex. Nat Neurosci 18:97-103.

Wang Y, Zhang B, Tao X, Wensveen JM, Smith EL, Chino YM (2017) Noisy spiking in visual area V2 of amblyopic monkeys. J Neurosci 37:922-935.

Wässle H, Boycott BB, Illing RB (1981) Morphology and mosaic of on- and off-beta cells in the cat retina and some functional considerations. Proc R Soc Lond B Biol Sci 212:177-195.

Whitney D, Levi DM (2011) Visual crowding: a fundamental limit on conscious perception and object recognition. Trends Cogn Sci 15:160-168.

Wiesel TN, Hubel DH (1963) Single-cell responses in striate cortex of kittens deprived of vision in one eye. J Neurophysiol 26:1003-1017.

Wool LE, Komban SJ, Kremkow J, Jansen M, Li X, Alonso JM, Zaidi Q (2015) Salience of unique hues and implications for color theory. J Vis 15(2):10 $1-11$.

Xing D, Yeh CI, Gordon J, Shapley RM (2014) Cortical brightness adaptation when darkness and brightness produce different dynamical states in the visual cortex. Proc Natl Acad Sci U S A 111:1210-1215.

Yeh CI, Xing D, Shapley RM (2009) "Black" responses dominate macaque primary visual cortex v1. J Neurosci 29:11753-11760.

Zahs KR, Stryker MP (1988) Segregation of ON and OFF afferents to ferret visual cortex. J Neurophysiol 59:1410-1429.

Zemon V, Gordon J, Welch J (1988) Asymmetries in ON and OFF visual pathways of humans revealed using contrast-evoked cortical potentials. Vis Neurosci 1:145-150.

Zhao L, Sendek C, Davoodnia V, Lashgari R, Dul MW, Zaidi Q, Alonso JM (2015) Effect of age and glaucoma on the detection of darks and lights. Invest Ophthalmol Vis Sci 56:7000-7006.

Zurawel G, Ayzenshtat I, Zweig S, Shapley R, Slovin H (2014) A contrast and surface code explains complex responses to black and white stimuli in V1. J Neurosci 34:14388-14402. 